\title{
UNCERTAINTY THROUGH THE LENSES OF A MIXED-FREQUENCY BAYESIAN PANEL MARKOV-SWITCHING MODEL
}

\author{
By Roberto CASARIN*, Claudia Foroni ${ }^{\dagger}$, MASSIMILIANO $^{*}$ \\ MARCELLINO $^{\ddagger}, \S$ AND FRANCESCO RAVAZZOLO ${ }^{\text {II, } \|}$ \\ University Ca' Foscari of Venice*, Deutsche Bundesbank ${ }^{\dagger}$, Bocconi University, \\ IGIER ${ }^{\ddagger}$, C.E.P.R. ${ }^{\S}$, Free University of Bozen/Bolzano ${ }^{\text {II }}$ and CAMP, BI Norwegian \\ Business School
}

\begin{abstract}
We propose a Bayesian panel model for mixed frequency data, where parameters can change over time according to a Markov process. Our model allows for both structural instability and random effects. To estimate the model, we develop a Markov Chain Monte Carlo algorithm for sampling from the joint posterior distribution, and we assess its performance in simulation experiments. We use the model to study the effects of macroeconomic uncertainty and financial uncertainty on a set of variables in a multi-country context including the US, several European countries and Japan. We find that there are large differences in the effects of uncertainty in the contraction regime and the expansion regime. The use of mixed frequency data amplifies the relevance of the asymmetry. Financial uncertainty plays a more important role than macroeconomic uncertainty, and its effects are also more homogeneous across variables and countries. Disregarding either the mixed-frequency component or the Markov-switching mechanism can bring to substantially different results.
\end{abstract}

1. Introduction. The role of uncertainty as a driver of macroeconomic fluctuations has been at the center of attention especially since the beginning of the Great Recession in 2007. Most of the literature has so far focused on measuring uncertainty and its effects in the US economy. Hence, there is a clear need to study whether the results for the US also hold for other countries, which differ for the structure of their goods, labour and financial markets, degree of openness, conduct of fiscal and monetary policy, and other institutional characteristics.

In this paper we take a multi-country perspective and assess the effects of uncertainty on different macroeconomic variables in various countries: US, Canada, Japan, Euro area as a whole and its main member States, UK, Switzerland, Norway, Sweden, all the countries for which we can construct a measure of uncertainty based on the Consensus Economic Forecasts. To properly address this question, we take a panel approach, as an unrestricted model for many variables and countries would be too large. We also allow for different effects of uncertainty over

Received January 2017; revised April 2018.

Key words and phrases. Dynamic panel models, econometrics, mixed-frequency, Markovswitching, Bayesian inference, Markov chain Monte Carlo. 
time, and in particular in expansionary and recessionary times. Finally, we exploit the presence of mixed frequency data to improve estimation efficiency and reduce identification problems; see, for example, Foroni and Marcellino (2014). A multicountry study on uncertainty has been recently proposed by Baker, Bloom and Davis (2016). However, the main focus of their work is to develop a new index of economic policy uncertainty (EPU) and the focus on a panel of countries is limited. To conduct this study, we develop a multi-country panel Markov-switching unrestricted mixed-data sampling regression (panel MS-UMIDAS from now on). Our first main contribution is therefore methodological. The panel MS-UMIDAS framework allows us to model a large panel of countries and several variables for each country, to use a time-varying transition mechanism and to model nonlinearities. Finally, it makes possible to consider variables at mixed frequencies.

The model is at the crossing of different strands of literature. Markov-switching dynamic panel models have been introduced by Kaufmann (2010), and further extended by Kaufmann (2015) to allow for a time-varying Markov-switching transition distribution, and by Billio et al. (2016b) to a multivariate VAR setup. Our model builds on Kaufmann $(2010,2015)$ and extends her model in two directions. First, we introduce country-specific and variable-specific random effects that allow us to obtain heteroskedastic effects with time-variation in the error variance. This is particularly relevant because it means that we do not need to include a Markovswitching mechanism in the variance once it is present in the random effects, in order to have time-variation in the variance of the residuals. Second, our model allows to use data at different frequencies.

There is an increasing literature on mixed frequency data. Here, we focus on one of the main strands, the mixed-data sampling (MIDAS) model. MIDAS regressions in their original specification, as introduced by Ghysels, Santa-Clara and Valkanov (2005), are tightly parameterized reduced form equations, which use distributed lag polynomials to parsimoniously incorporate high-frequency information into models for low-frequency variables. Foroni, Marcellino and Schumacher (2015) show that an unrestricted variant of MIDAS, which does not resort to functional distributed lag polynomials and preserves linearity of the model (UMIDAS), is particularly suited when the frequency mismatch is not too big, as in the case of macroeconomic data. Since we are interested in modelling macroeconomic variables, and due to the simplicity of the UMIDAS approach, we adopt this specification in our panel MS context. We highlight that the use of different MIDAS parameterizations [Ghysels, Santa-Clara and Valkanov (2005), Ghysels, Sinko and Valkanov (2007), Pettenuzzo, Timmermann and Valkanov (2016)] is allowed within our framework and does not pose any additional conceptual difficulty. It would imply though an enlargement of the parameter space and the addition of a step in the estimation algorithm.

Our paper relates also to other contributions in the mixed-frequency literature. Guérin and Marcellino (2013) introduce Markov-switching MIDAS and apply this model to the prediction of the US economic activity. Khalaf et al. (2013) have 
extended the MIDAS approach to the panel regression models suitable for analysis with GMM methods. We extend both these papers because we introduce here a Markov-switching panel MIDAS.

The estimation of our model is conducted in a Bayesian framework. In order to avoid overparameterization issues and overfitting problems we follow a hierarchical strategy in the specification of the prior as suggested in the Bayesian dynamic panel modeling literature [Canova and Ciccarelli (2004, 2009), Bassetti, Casarin and Leisen (2014), Kaufmann (2010)]. The hierarchical prior can be used to incorporate cross-equation interdependencies and various degrees of information pooling across countries [Chib and Greenberg (1995), Min and Zellner (1993)], and allows us to naturally introduce random effects into the panel model. Although the MIDAS models have been typically used in a classical estimation context, recently the literature has expanded into the Bayesian estimation of this class of models [Foroni, Ravazzolo and Ribeiro (2015), Pettenuzzo, Timmermann and Valkanov (2016), Rodriguez and Puggioni (2010)].

As a second main contribution, we apply our model to study the effects of uncertainty on different sectors and variables across a panel of developed countries, including data at different frequencies and at the same time allowing for different regimes. In our analysis, we consider different measures of uncertainty available in the literature: a measure of forecast disagreement as proxy for macroeconomic uncertainty, as in Dovern, Fritsche and Slacalek (2012), and the VIX as proxy of financial uncertainty, as proposed by Bloom (2009) in his seminal paper. We assume that monthly uncertainty is exogenous to quarterly macroeconomic variables. The identification scheme relies on the release time of the uncertaintyrelated and macroeconomic variables, with the former generally released earlier and in higher frequency and the latter released with some delay and in low frequency. The literature has documented evidence of substantial time-variation in the transmission and implications of uncertainty shocks [Alessandri and Mumtaz (2014), Caggiano, Castelnuovo and Groshenny (2014), Mumtaz and Theodoridis (2018)]. Our Markov-switching specification can capture this type of behavior. It also partially protects from omitted variable bias, as potential unmodelled variables which affect both macroeconomic variables and uncertainty are captured by the regime switching mechanism.

Our empirical results can be summarized as follows. There are large differences in the effects of uncertainty in the contraction regime and the expansion regime. The use of mixed frequency data rather than quarterly uncertainty variables amplifies the relevance of the asymmetry. Moreover, financial uncertainty plays a more important role than macroeconomic uncertainty. Its effects are stronger in the contraction regime than in the expansion regime, in particular on the real variables. Further, the effects of financial uncertainty are also more homogeneous across variables and countries than those of macroeconomic uncertainty. When financial uncertainty is removed, the role of macroeconomic uncertainty increases, capturing part of the financial uncertainty and highlighting the need of jointly considering both types of uncertainty to avoid biased results, in line with the results 
in Jurado, Ludvigson and $\mathrm{Ng}$ (2015) and Carriero, Clark and Marcellino (2018). Finally, we show that disregarding either the mixed-frequency component or the Markov-switching mechanism can bring to substantially different results.

This paper is organized as follows. Section 2 presents our Bayesian panel Markov-switching MIDAS model, discusses the Bayesian inference framework and confirms efficiency and convergence of our estimation method. Section 3 presents empirical results on the effects of financial and macroeconomic uncertainty on macroeconomic variables. Section 4 concludes. Proofs of the results and additional details are presented in a set of Appendices. Details on the estimation algorithm and additional empirical results can be found in the supplementary materials Supplement B-Supplement E [Casarin et al. (2018)].

2. A panel Markov-switching UMIDAS model. We introduce a panel Markov-switching UMIDAS specification, with which we model a set of countries and variables and, at the same time, we allow to use a time-varying transition mechanism and to exploit data at different frequency.

The idea of a Markov-switching dynamic panel model has been introduced by Kaufmann (2010), who considers a group-specific time series model, in which the information about the business cycle turning points is extracted by estimating groups of series that follow a similar time series process over time. The parameters that describe the process are shrunk towards group-specific means rather than pooled, allowing for group-specific parameter heterogeneity. The different groups of series are then linked by a dynamic structure. Kaufmann (2015) extends her previous model to allow for endogenous transition probabilities.

Our model builds on Kaufmann $(2010,2015)$. We consider a panel of $G$ countries and $n_{g}$ variables for each country, and extend the previous studies in two directions. First, we apply country- and variable-specific random effects that allow us to obtain heteroskedastic effects with time-variation in the error variance without the requirement of specifying a dynamic model for it. Second, our model allows to use data at different frequencies, without the need of previously aggregating them to the lowest available frequency.

2.1. Model specification. Let us consider a panel of $G$ countries and $n_{g}$ variables for each country. The number of variables for each country does not have to be the same. However, to keep the notation simple, we will consider, with no loss of generality, each country having $n_{g}$ variables.

The panel specification we propose is the following:

$$
c_{i g}\left(L^{m}, s_{g t}\right) y_{i g t}=\mu_{i g}\left(s_{g t}\right)+\sum_{j=1}^{N} \delta_{i g j}\left(L, s_{g t}\right) x_{i g j t}+\varepsilon_{i g t},
$$

for all $i$ and $g$, where $\varepsilon_{i g t} \stackrel{\text { i.i.d. }}{\sim} \mathcal{N}\left(0, \sigma_{i g}^{2}\right)$ for all $t$ and the errors are uncorrelated across equations at all leads and lags. 
The lag structure of each variable $y_{i g t}$ and of the covariates $x_{i g j t}$ are defined respectively as:

$$
c_{i g}\left(L^{m}, s_{g t}\right)=1-\sum_{l=1}^{c} L^{m l} c_{i g l}\left(s_{g t}\right), \quad \delta_{i g j}\left(L, s_{g t}\right)=\sum_{d=0}^{v} \delta_{i g j d}\left(s_{g t}\right) L^{d},
$$

where $L$ is the lag operator. As $y_{i g t}$ are sampled at frequency $m$, the lag operator in $c_{i g}\left(L^{m}, s_{g t}\right)$ is a power of $L^{m}$, with $L^{m} y_{i g t}=y_{i g t-m}$ and $c$ is the maximum lag length for the variable $y_{i g t}$. The variables $x_{i g j t}$ are instead sampled at each $t$, so that we do not need to restrict $L$ in $\delta_{i g j}\left(L, s_{g t}\right)$ and $v$ is the maximum lag length for the covariate $x_{i g j t}$. Hence, each variable $y_{i g t}$ is influenced by its own lags, and by the contemporaneous and lagged values of all the covariates $x_{i g j t}$, with $j=1, \ldots, N$, and the coefficients can vary depending on $s_{g t}$. The variable $s_{g t}$ is a Markov chain process, with $t=m, 2 m, \ldots, m T_{q}$, and it has a transition probability

$$
P\left(s_{g t}=k \mid s_{g t-m}=h\right)=p_{g h k},
$$

with $h, k=1, \ldots, K$, where $K$ is the total number of regimes. The switching coefficients for each country $g=1, \ldots, G$ and variable $i=1, \ldots, n_{g}$ are defined as

$$
\begin{aligned}
& \mu_{i g}\left(s_{g t}\right)=\sum_{k=1}^{K} \xi_{g k t} \mu_{i g k}, \\
& c_{i g l}\left(s_{g t}\right)=\sum_{k=1}^{K} \xi_{g k t} c_{i g l k}, \delta_{i j d g}\left(s_{g t}\right)=\sum_{k=1}^{K} \xi_{g k t} \delta_{i j d g k},
\end{aligned}
$$

for $l=1, \ldots, c$ and $j=1, \ldots, N$ where $\xi_{g k t}$ is the indicator function of being in a specific regime $k$ and it is defined as $\xi_{g k t}=\mathbb{I}_{\{k\}}\left(s_{g t}\right)$.

The model in equation (2.1) is quite general, since it assumes that the dependent variables are observed at the same $(m=1)$ or lower $(m>1)$ frequency than the independent variables. Further, the specification can be easily extended to allow for dynamic interaction effects between the variables of each country and also between variables of different countries. Unfortunately, in practice the interaction effects would lead to a larger number of parameters to estimate and to a potential overfitting problem. Therefore, we leave the modelling of these effects for further research.

2.2. A hierarchical prior specification. We follow Canova and Ciccarelli (2004, 2009) and assume a hierarchical prior on the switching coefficients. The hierarchical prior specification is particularly suited in this context where the number of parameters to estimate is large. While the estimation of all the parameters can lead to overfitting problems, the use of restrictions, such as parameter pooling, can be a strong assumption conducting to misleading results and poor forecasting 
performance. The hierarchical specification allows instead for different degrees of information pooling across countries and series, by assuming conditional independence across countries and series and by introducing panel- and country-specific common factors [Canova and Ciccarelli (2004, 2009), Bassetti, Casarin and Leisen (2014), Billio et al. (2016b), Koop and Korobilis (2016)]. Also, this motivates the use of panel models with random effects instead of using pooling or equation-byequation estimation.

In detail, we design the prior distributions in a way that the regime-specific coefficients of the different time series are shrunk toward country-specific and regimespecific common means:

$$
\begin{aligned}
\mu_{i g k}= & \mu_{k}+\zeta_{\mu, g k}+\eta_{\mu, i g k}, \\
& \zeta_{\mu, g k} \sim \mathcal{N}\left(0, r_{\mu, k}\right), \eta_{\mu, i g k} \sim \mathcal{N}\left(0, q_{\mu, g k}\right), \\
c_{i g l k}= & c_{l k}+\zeta_{c, g l k}+\eta_{c, i g l k}, \\
& \zeta_{c, g l k} \sim \mathcal{N}\left(0, r_{c, k}\right), \eta_{c, i g l k} \sim \mathcal{N}\left(0, q_{c, g k}\right), \\
\delta_{i g j d k}= & \delta_{j d k}+\zeta_{\delta, g j d k}+\eta_{\delta, i g j d k}, \\
& \zeta_{\delta, g j d k} \sim \mathcal{N}\left(0, r_{\delta, k}\right), \eta_{\delta, i g j d k} \sim \mathcal{N}\left(0, q_{\delta, g k}\right),
\end{aligned}
$$

with $\operatorname{Cov}\left(\eta_{\mu, i g k}, \eta_{c, i^{\prime} g^{\prime} l k^{\prime}}\right)=0, \operatorname{Cov}\left(\eta_{\mu, i g k}, \eta_{\delta, i^{\prime} g^{\prime} j d k^{\prime}}\right)=0$, and $\operatorname{Cov}\left(\eta_{c, i g l k}\right.$, $\left.\eta_{\delta, i^{\prime} g^{\prime} j d k^{\prime}}\right)=0$, for all $i, i^{\prime}, g, g^{\prime}, j, l, d$ and $k, k^{\prime}$. The country- and regime-specific random effects are $\zeta_{\mu, g k}, \zeta_{c, g l k}$ and $\zeta_{\delta, g j d k}$. Our choice of the hierarchical prior distribution depends mainly on the application. In our empirical exercise we believe that the impact of uncertainty might substantially differ across countries, but that variables in the same country will respond similarly with a larger negative effect in the contraction regime than in the expansion regime. This motivates our choice for country- and regime-specific random effects. An alternative modelling strategy is to shrink the coefficients towards a variable-specific mean, $\zeta_{\mu, i k}$, instead of a country-specific mean, $\zeta_{\mu, g k}$. This can be obtained by setting $\mu_{i g k}=\mu_{k}+\zeta_{\mu, i k}+\eta_{\mu, i g k}, \eta_{\mu, i g k} \sim \mathcal{N}\left(0, q_{\mu, g k}\right), \zeta_{\mu, i k} \sim \mathcal{N}\left(0, r_{\mu, k}\right)$. A similar model can be used for $c_{i g l k}$ and $\delta_{i g j d k}$. In cases for which the choice among different priors is controversial (e.g., in the cases in which the hierarchical prior changes over time), Koop and Korobilis (2016) develop an approach which allows for dynamic model averaging or selection of models with different hierarchical priors.

In order to complete the elicitation of the hierarchical prior distribution, we assume a truncated normal prior distribution for the common intercepts $\boldsymbol{\mu}=$ $\left(\mu_{1}, \ldots, \mu_{K}\right)^{\prime}$ :

$$
\boldsymbol{\mu} \sim \mathcal{N}_{K}\left(\mathbf{0}_{K}, s_{0}^{2} I_{K}\right) \mathbb{I}_{\mathcal{A}_{\mu}}(\boldsymbol{\mu}),
$$

where $\mathbf{0}_{K}$ is the $K$-dimensional null vector, $I_{K}$ the $K$-dimensional identity matrix, $s_{0}$ is an hyperparameter, and $\mathcal{A}_{\mu}$ is the set of all possible values of $\boldsymbol{\mu}$ which satisfy 
some identification constraints, such as $\mu_{1}<\cdots<\mu_{K}$. We refer to FrühwirthSchnatter (2006), ch. 3-4, for an introduction to the problems of regime identification and label switching and to Billio et al. (2016b) and Billio et al. (2012) for the use of these constraints in business cycle analysis. Alternative identification constraints can be used. For example, if one expects the duration of the states to be different, then constraints on the transition matrix can be employed to effectively identify the regimes. As initial condition, in the application we set $s_{0}=10$.

We assume cross-regime independent normal prior distributions for the common coefficients $\mathbf{c}_{k}=\left(c_{1 k}, \ldots, c_{c k}\right)^{\prime}$ and $\boldsymbol{\delta}_{k}=\left(\delta_{10 k}, \ldots, \delta_{1 N k}, \ldots, \delta_{v 0 k}, \ldots\right.$, $\left.\delta_{v N k}\right)^{\prime}$ :

$$
\mathbf{c}_{k} \stackrel{\text { i.i.d. }}{\sim} \mathcal{N}_{c}\left(\mathbf{0}_{c}, r_{0}^{2} I_{c}\right), \quad \boldsymbol{\delta}_{k} \stackrel{\text { i.i.d. }}{\sim} \mathcal{N}_{N(v+1)}\left(\mathbf{0}_{N(v+1)}, r_{0}^{2} I_{N(v+1)}\right),
$$

for $k=1, \ldots, K$. In the empirical analysis we set the hyper-parameter $r_{0}$ to 10 .

We assume cross-regime independent inverse gamma prior distributions for the two sets of scale hyper-parameters $q_{\mu, g k}, q_{c, g k}, q_{\delta, g k}$ and $r_{\mu, k}, r_{c, k}, r_{\delta, k}$ of the panel coefficients in equation (2.4) to (2.6):

$$
q_{\mu, g k}, q_{c, g k}, q_{\delta, g k} \stackrel{\text { i.i.d. }}{\sim} \mathcal{I} \mathcal{G}\left(n_{0}, m_{0}\right), \quad r_{\mu, k}, r_{c, k}, r_{\delta, k} \stackrel{\text { i.i.d. }}{\sim} \mathcal{I} \mathcal{G}\left(n_{0}, m_{0}\right),
$$

for $k=1, \ldots, K$, and $g=1, \ldots, G$, where $\mathcal{I} \mathcal{G}\left(n^{\prime}, m^{\prime}\right)$ denotes the inverse gamma distribution with shape and rate parameters $n^{\prime}$ and $m^{\prime}$ and density function given in Supplement B. In the empirical exercises we set the hyper-parameters $n_{0}$ and $m_{0}$ equal to 1.

As regards the scale parameter of the error term in equation (2.1), we also consider country- and variables specific components, that is we define our prior as:

$$
\sigma_{i g}^{2}=\sigma^{2} \lambda_{i g}^{-1} \chi_{g}^{-1} \text {, }
$$

for $g=1, \ldots, G$, and $i=1, \ldots, n_{g}$, where $\sigma^{2}$ is a common scaling factor, $\chi_{g}$ is a country-specific factor which captures the potential cross-country variance heterogeneity, and $\lambda_{i g}$ is a variable-specific scale factor. We use the following inverted gamma and gamma hierarchical prior distributions, which are usually assumed for the scale parameters in multi-country panel models [see, e.g., Bassetti, Casarin and Leisen (2014) and references therein]

$$
\sigma^{2} \stackrel{\text { i.i.d. }}{\sim} \mathcal{I} \mathcal{G}\left(a_{0}, b_{0}\right), \quad \lambda_{i g} \stackrel{\text { i.i.d. }}{\sim} \mathcal{G} a\left(c_{10}, d_{10}\right), \quad \chi_{g} \stackrel{\text { i.i.d. }}{\sim} \mathcal{G} a\left(c_{20}, d_{20}\right),
$$

for $g=1, \ldots, G$ and $i=1, \ldots, n_{g}$, where $\mathcal{G} a\left(c^{\prime}, d^{\prime}\right)$ denotes the gamma distribution with shape and rate parameters $c^{\prime}$ and $d^{\prime}$, respectively and density function given in Supplement B. In the empirical exercises we use the hyper-parameters: $a_{0}=1, b_{0}=1, a_{10}=1, b_{10}=1, c_{20}=1$ and $d_{20}=1$.

We assume independent hierarchical Dirichlet prior distributions for the rows of the country-specific transition probabilities. Let $\mathbf{p}_{g k}=\left(p_{g, k 1}, \ldots, p_{g, k K}\right)^{\prime}$ and $\boldsymbol{v}_{k}=\left(v_{k 1}, \ldots, v_{k K}\right)^{\prime}$, then our prior distribution is

$$
\mathbf{p}_{g k} \stackrel{\text { i.i.d. }}{\sim} \operatorname{Dir}\left(\phi v_{1}, \ldots, \phi v_{K}\right), \quad \boldsymbol{v}_{k} \stackrel{\text { i.i.d. }}{\sim} \operatorname{Dir}(1 / K, \ldots, 1 / K),
$$

for $k=1, \ldots, K$ and $g=1, \ldots, G$, where $\phi=\sum_{h=1}^{K} v_{h}$. 
2.3. A random effect model. Under the hierarchical prior specification that we elicited in the previous subsection, the dynamic panel model can be reinterpreted as a random effect model with country-specific and regime-specific effects for intercepts, regression coefficients and scale parameters. By replacing the coefficients in equation (2.1) with the switching representation in equation (2.2) and the hierarchical prior structure in equation (2.4) to (2.6), and rearranging terms, we obtain the following model:

$$
\begin{aligned}
y_{i g t}= & \sum_{k=1}^{K} \xi_{g k t}\left\{\left(\mu_{k}+\zeta_{\mu, g k}+\eta_{\mu, i g k}\right)+\sum_{l=1}^{c} y_{i g t-m l}\left(c_{l k}+\zeta_{c, g l k}\right.\right. \\
& \left.\left.+\eta_{c, i g l k}\right)+\sum_{j=1}^{N} \sum_{d=0}^{v} x_{i g j t-d}\left(\delta_{j d k}+\zeta_{\delta, g j d k}+\eta_{\delta, i g j d k}\right)\right\}+\varepsilon_{i g t},
\end{aligned}
$$

for $t=m, 2 m, \ldots, T_{q} m$. The model in equation (2.7) can be regarded as the Markov-switching extension of a panel MIDAS model such as the one discussed in Khalaf et al. (2013).

While the expanded representation of our Bayesian panel MS-UMIDAS model as in equation (2.1) is useful to understand how the random effects enter into the model, it can result uneasy for the presentation of the inference procedure. Therefore, we rewrite equation (2.7) in a more concise form, combining the MIDAS representation [Pettenuzzo, Timmermann and Valkanov (2016)] with a compact switching regression representation [Billio et al. (2016b)].

For this purpose, define the allocation variable vector $\xi_{g t}=\left(\xi_{g 1 t}, \ldots, \xi_{g K t}\right)^{\prime}$, where $\xi_{g k t}$ is the indicator function of being in a specific regime. Further, we define the autoregressive component $\mathbf{v}_{i g t}=\left(y_{i g t-m}, \ldots, y_{i g t-m c}\right)^{\prime}$ and $\mathbf{x}_{i g, j t}=$ $\left(x_{i g}, j t, \ldots, x_{i g, j t-v}\right)^{\prime}$ which includes the contemporaneous and lagged values of the $j$ th exogenous variable, for $j=1, \ldots, N$. In addition, we collect all the parameters into vectors. First, we introduce vectors, of regime-specific common factors for intercepts, $\boldsymbol{\mu}=\left(\mu_{1}, \ldots, \mu_{K}\right)^{\prime}$, autoregressive coefficients, $\mathbf{c}_{l}=$ $\left(c_{l 1}, \ldots, c_{l K}\right)^{\prime}$, for $l=1, \ldots, c$, and contemporaneous and lagged covariate coefficients $\boldsymbol{\delta}_{j d}=\left(\delta_{j d 1}, \ldots, \delta_{j d K}\right)^{\prime}$, for $j=1, \ldots, N$ and $d=0, \ldots, v$. Second, we define the regime- and country-specific common factors, $\zeta_{\mu, g}=\left(\zeta_{\mu, g 1}, \ldots, \zeta_{\mu, g}\right)^{\prime}$, $\zeta_{c, g l}=\left(\zeta_{c, g l 1}, \ldots, \zeta_{c, g l K}\right)^{\prime}, \zeta_{\delta, g j d}=\left(\zeta_{\delta, g j d 1}, \ldots, \zeta_{\delta, g j d K}\right)^{\prime}$, for $g=1, \ldots, G$, $l=1, \ldots, c$ and $j=1, \ldots, N$. Finally, for $i=1, \ldots, n_{g}$ and $g=1, \ldots, G$, the random effects are collected into the vectors $\boldsymbol{\eta}_{\mu, i g}=\left(\eta_{\mu, i g 1}, \ldots, \eta_{\mu, i g K}\right)^{\prime}, \boldsymbol{\eta}_{c, i g l}=$ $\left(\eta_{c, i g l 1}, \ldots, \eta_{c, i g l K}\right)^{\prime}$, and $\eta_{\delta, i g j d}=\left(\eta_{\delta, i g j d 1}, \ldots, \eta_{\delta, i g j d K}\right)^{\prime}$, for $l=1, \ldots, c, j=$ $1, \ldots, N$ and $d=0, \ldots, v$.

The following result holds.

PROPOSITION 1. The model in equation (2.7) can be written as

$$
y_{i g t}=\mathbf{z}_{i g t}^{\prime}\left(\boldsymbol{\beta}+\zeta_{g}+\eta_{i g}\right)+\varepsilon_{i g t},
$$


with $\boldsymbol{\beta}=\left(\boldsymbol{\mu}, \mathbf{c}_{1}, \ldots, \mathbf{c}_{c}, \boldsymbol{\delta}_{10}, \ldots, \boldsymbol{\delta}_{N v}\right)^{\prime}$ the parameter vector, $\mathbf{z}_{i g t}=\left(\boldsymbol{\xi}_{g t}^{\prime}, \mathbf{v}_{i g t}^{\prime} \otimes\right.$ $\left.\xi_{g t}^{\prime}, \mathbf{x}_{i g, 1 t}^{\prime} \otimes \xi_{g t}^{\prime}, \ldots, \mathbf{x}_{i g, N t}^{\prime} \otimes \xi_{g t}^{\prime}\right)^{\prime}$ the covariate vector of dimension $K(1+c+$ $N(v+1)) \times 1, \boldsymbol{\eta}_{i g}=\left(\boldsymbol{\eta}_{\mu, i g}, \boldsymbol{\eta}_{c, i g 1}, \ldots, \boldsymbol{\eta}_{c, i g c}, \boldsymbol{\eta}_{\delta, i g 10}, \ldots, \boldsymbol{\eta}_{\delta, i g N v}\right)^{\prime}$ the variablespecific random effects vector, and $\zeta_{g}=\left(\zeta_{\mu g}, \zeta_{c, g 1}, \ldots, \zeta_{c, g c}, \zeta_{\delta, g 10}, \ldots, \zeta_{\delta, g N v}\right)^{\prime}$ the country-specific random effects vector.

\section{Proof. See Appendix A.}

2.4. Posterior approximation. The joint posterior distribution obtained from the likelihood and the priors outlined in the previous subsection is not tractable, thus our Bayesian inference approach relies on a Markov chain Monte Carlo (MCMC) approximation of the posterior distribution [Robert and Casella (1999)]. At each iteration, the MCMC algorithm generates a random sample for the latent variables and the parameters of the model from their full conditional distributions. The sequence of MCMC samples obtained after removing the initial set of burn-in samples can be used to approximate any posterior quantity of interest.

We design an efficient MCMC algorithm by combining a multi-move strategy, that is by sampling jontly blocks of parameters and latent variables, with a RaoBlackwellization strategy, that is, by integrating out analytically some of the conditioning variables [Liu (1994), Roberts and Sahu (1997)].

In detail, let $\mathbf{y}=\left(\mathbf{y}_{m(c+1)}^{\prime}, \ldots, \mathbf{y}_{m T_{q}}^{\prime}\right)^{\prime}$ be the vector of observations, with $\mathbf{y}_{t}=\left(\mathbf{y}_{1 t}^{\prime}, \ldots, \mathbf{y}_{G t}^{\prime}\right)^{\prime}, \mathbf{y}_{g t}=\left(y_{1 g t}, \ldots, y_{n_{g} g}\right)^{\prime}, \boldsymbol{\xi}=\left(\boldsymbol{\xi}_{1}^{\prime}, \ldots, \boldsymbol{\xi}_{G}^{\prime}\right)^{\prime}$ the allocation variable, with $\boldsymbol{\xi}_{g}=\left(\boldsymbol{\xi}_{g, m(c+1)}^{\prime}, \ldots, \boldsymbol{\xi}_{g, m T_{q}}^{\prime}\right)^{\prime}, \boldsymbol{\eta}_{i g k}=\left(\eta_{\mu, i g k}, \eta_{c, i g k}, \eta_{\delta, i g k}\right)^{\prime}, \boldsymbol{\zeta}=$ $\left(\zeta_{1}^{\prime}, \ldots, \zeta_{G}^{\prime}\right)^{\prime}, \boldsymbol{\eta}=\left(\eta_{11}^{\prime}, \ldots, \eta_{n_{G} G}^{\prime}\right)^{\prime}$. Then, the complete likelihood of the model in equation (2.8) is

$$
L(\mathbf{y}, \boldsymbol{\xi} \mid \boldsymbol{\theta}, \boldsymbol{\zeta}, \boldsymbol{\eta})=\prod_{t \in \mathcal{T}} \prod_{g=1}^{G} \prod_{i=1}^{n_{g}}\left(2 \pi \sigma_{i g}^{2}\right)^{-1 / 2} \exp \left\{-\frac{\varepsilon_{i g t}^{2}}{2 \sigma_{i g}^{2}}\right\} \prod_{f=1}^{K} \prod_{k=1}^{K} p_{g f k}^{\xi_{g f t-1} \xi_{g k t}},
$$

where $p_{g f k}$ are the country-specific transition probabilities, $\varepsilon_{i g t}$ is defined in equation (A.3) as $\varepsilon_{i g t}=y_{i g t}-\mathbf{z}_{i g t}^{\prime}\left(\boldsymbol{\beta}+\zeta_{g}+\boldsymbol{\eta}_{i g}\right)$ in the proof of Proposition 1 in Appendix $\mathrm{A}, \mathbf{z}_{i g t}$ is defined in Proposition 1 , and $\mathcal{T}=\{m(c+$ $\left.1), m c+2 m, \ldots, m T_{q}\right\}$. Further, $\boldsymbol{\theta}=\left(\boldsymbol{\beta}, \sigma^{2}, \boldsymbol{\lambda}, \boldsymbol{\chi}, \mathbf{q}, \mathbf{p}\right)$ indicates the parameter vector, with $\lambda=\left(\lambda_{11}, \ldots, \lambda_{n_{G} G}\right)^{\prime}, \chi=\left(\chi_{1}, \ldots, \chi_{G}\right)^{\prime}, \mathbf{q}=\left(\mathbf{q}_{11}^{\prime}, \ldots, \mathbf{q}_{G K}^{\prime}\right)^{\prime}$, $\mathbf{r}=\left(\mathbf{r}_{1}^{\prime}, \ldots, \mathbf{r}_{K}^{\prime}\right)^{\prime}$, where $\mathbf{q}_{g k}=\operatorname{diagrv}\left\{Q_{g k}\right\}, \mathbf{r}_{k}=\operatorname{diagrv}\left\{R_{k}\right\}, \operatorname{diagrv}\{R\}$ the operator which transforms the diagonal matrix $R$ into a vector, $\mathbf{p}=\left(\operatorname{vec}\left\{P_{1}\right\}\right.$, $\left.\ldots, \operatorname{vec}\left\{P_{G}\right\}\right), \operatorname{vec}\{R\}$ the operator which stacks vertically the columns of the ma$\operatorname{trix} R, Q_{g k}=\operatorname{diag}\left\{\left(q_{\mu, g k}, \iota_{c}^{\prime} \otimes q_{c, g k}, \iota_{N(v+1)}^{\prime} \otimes\left(q_{\delta, g k}\right)\right\}, R_{k}=\operatorname{diag}\left\{\left(r_{\mu, k}, \iota_{c}^{\prime} \otimes r_{c, k}\right.\right.\right.$, $\left.\iota_{N(v+1)}^{\prime} \otimes\left(r_{\delta, k}\right)\right\}, \operatorname{diag}\{\mathbf{r}\}$ the operator which transforms the vector $\mathbf{r}$ into a diagonal matrix with the elements of $\mathbf{r}$ on the main diagonal, and $P_{g}^{\prime}=\left(\mathbf{p}_{g 1}, \ldots, \mathbf{p}_{g K}\right)$. 
Then, the posterior is

$$
\begin{aligned}
\pi(\boldsymbol{\xi}, \zeta, \boldsymbol{\eta}, \boldsymbol{\theta} \mid \mathbf{y}) \propto & L(\mathbf{y}, \boldsymbol{\xi} \mid \boldsymbol{\theta}, \boldsymbol{\zeta}, \boldsymbol{\eta}) \pi\left(\sigma^{2}\right) \prod_{k=1}^{K} \pi\left(\mu_{k}\right) \pi\left(\mathbf{c}_{k}\right) \pi\left(\boldsymbol{\delta}_{k}\right) \pi\left(\boldsymbol{R}_{k}\right) \pi\left(\boldsymbol{v}_{k}\right) \\
& \times \prod_{g=1}^{G} \pi\left(\chi_{g}\right) \pi\left(\boldsymbol{\zeta}_{g k}\right) \pi\left(Q_{g k}\right) \pi\left(\mathbf{p}_{g k}\right) \prod_{i=1}^{n_{g}} \pi\left(\lambda_{i g}\right) \pi\left(\boldsymbol{\eta}_{i g k}\right),
\end{aligned}
$$

which is not analytically tractable. Thus, we apply a Gibbs sampler, which iterates over the following steps:

(i) Draw $\boldsymbol{\zeta}, \boldsymbol{\eta}, \boldsymbol{\xi}, \boldsymbol{\beta}, P_{1}, \ldots, P_{G}, \boldsymbol{v}$ from $p\left(\boldsymbol{\zeta}, \boldsymbol{\eta}, \boldsymbol{\xi}, \boldsymbol{\beta}, \mathbf{p}, \boldsymbol{v} \mid \sigma^{2}, \boldsymbol{\lambda}, \boldsymbol{\chi}, \mathbf{q}, \mathbf{r}, \mathbf{y}\right)$.

(ii) Draw $\sigma^{2}, \lambda, \chi, \mathbf{q}, \mathbf{r}$ from $p\left(\sigma^{2}, \lambda, \chi, \mathbf{q}, \mathbf{r} \mid \boldsymbol{\beta}, \mathbf{p}, \boldsymbol{v}, \boldsymbol{\zeta}, \boldsymbol{\eta}, \boldsymbol{\xi}, \mathbf{y}\right)$.

The following result applies for step (i) of the Gibbs and shows that the model in equation (2.9) naturally exhibits heteroskedastic effects driven by the covariates and the Markov-switching process.

PROPOSITION 2. Marginalizing out the random effects in the right-hand side of equation (2.8) one obtains

$$
\begin{aligned}
y_{i g t}= & \mathbf{z}_{i g t}^{\prime} \boldsymbol{\beta}+\tilde{\varepsilon}_{i g t}, \\
& \tilde{\varepsilon}_{i g t} \sim \mathcal{N}\left(0, \sigma_{i g t}^{2}\right), t=m(c+1), 2 m, \ldots, m T_{q},
\end{aligned}
$$

with $\sigma_{i g t}^{2}=\sigma^{2} \lambda_{i g}^{-1} \chi_{g}^{-1}+\mathbf{z}_{i g t}^{\prime}\left(R+Q_{g}\right) \mathbf{z}_{i g t}$.

ProOF. See Appendix A.

Proposition 2 has two important consequences, one for the efficiency of the posterior approximation algorithm and the other for the properties of the model. First, since the random effect parameters can be integrated out of the likelihood (Rao-Blackwellization), and the time-varying error variance remains linear in the parameter $\boldsymbol{\beta}$, conditionally to the hidden Markov chain, we can sample from the full conditional distribution of $\beta, P_{1}, \ldots, P_{G}, \boldsymbol{v}$ and $\xi$ with the conditioned variables $\zeta$ and $\eta$ integrated out. This motivates the use of the following sampling scheme, known as collapsed Gibbs [Kaufmann (2010)], for the Step (i):

(i.1) Draw $\boldsymbol{\beta}, P_{1}, \ldots, P_{G}, \boldsymbol{v}, \boldsymbol{\xi}$ from $p\left(\boldsymbol{\beta}, \mathbf{p}, \boldsymbol{v}, \boldsymbol{\xi} \mid \sigma^{2}, \lambda, \chi, \mathbf{q}, \mathbf{r}, \mathbf{y}\right)$.

(i.2) Draw $\zeta, \eta$ from $p\left(\zeta, \boldsymbol{\eta} \mid \boldsymbol{\beta}, \mathbf{p}, \boldsymbol{v}, \sigma^{2}, \boldsymbol{\lambda}, \boldsymbol{\chi}, \mathbf{q}, \mathbf{r}, \boldsymbol{\xi}, \mathbf{y}\right)$.

The main advantage of this Rao-Blackwellization and collapsed Gibbs sampling is that it accelerates convergence to the target distribution. In the derivation of the full conditional distribution in the step (i.1) we use the complete data likelihood of the model in equation (2.9). Each step of the Gibbs sampler is blocked further. 
The details of the derivation of the full conditional distributions and the sampling methods used are given in Supplement B.

The second implication of Proposition 2, is that we do not need to include a Markov-switching mechanism in the variance. In time series models without random effects, time-varying volatility is usually included by specifying a dynamic model for the conditional variance. However, in our dynamic panel model the Markov-switching dynamics is included in the random effects, and since they are usually integrated out in model fitting and forecasting, this implies (as shown in Proposition 2) that time-varying variance is naturally included in the analysis.

Our proposed MCMC method for the posterior approximation is efficient and it reaches convergence to the true parameters. In Supplement $\mathrm{C}$ we report the results of a simulation study, in which we show that the MCMC chain is converging, and that latent Markov-switching regimes and parameter values are precisely estimated.

3. Economic uncertainty in a panel of countries. We apply our panel Markov-switching UMIDAS model to study the effects of uncertainty on different sectors and variables across a panel of countries. After a brief overview of the main contributions in the uncertainty literature, we describe the dataset and the measures of uncertainty that we consider, comment on the main empirical results, and present some robustness analyses. Further, we show that disregarding either the mixed-frequency component or the Markov-switching mechanism, key ingredients of our panel MS-UMIDAS specification, can bring to substantially different results.

3.1. Uncertainty and macroeconomic effects. The interest in uncertainty has grown enormously over the recent years. Since the seminal paper of Bloom (2009), research has focused on creating new approaches to measure uncertainty and its effects. Bloom (2009) himself defines his measure of uncertainty as the unconditional volatility of stock market returns. Baker, Bloom and Davis (2016) develop an index of economic policy uncertainty which reflects the frequency of uncertaintyrelated words in the articles of leading newspapers. Scotti (2016) proposes an uncertainty index which aims at capturing how uncertain agents are about the current real economic activity, using surprises from Bloomberg forecasts. Rossi and Sekhposyan (2015) create a macroeconomic uncertainty index based on comparing the realized forecast error of the real GDP growth with the historical forecast error distribution of the same variable. Jurado, Ludvigson and $\mathrm{Ng}$ (2015) and Carriero, Clark and Marcellino (2018) provide a measure of uncertainty based on whether a large set of macroeconomic and financial variables become more or less predictable.

Despite the different measures of uncertainty that have been proposed, studies agree that macroeconomic uncertainty is countercyclical. Here we sketch only some of the contributions to a voluminous and expanding literature. For a more 
extensive review see Bloom (2014). An increase in uncertainty is typically associated with large declines in real activity. Caggiano, Castelnuovo and Groshenny (2014) show that the impact of an uncertainty shock on unemployment in the US is much larger during recessions, suggesting a different behavior depending on the state of the economy. Carriero, Clark and Marcellino (2018) proposes an econometric framework for jointly measuring uncertainty and capturing its impact on the economy. The authors find sizeable effects of uncertainty on key macroeconomic variables. Ferrara and Guerin (2015) analyze the role of uncertainty for the US in a mixed-frequency set up and find that credit and labor market variables react the most to uncertainty shocks, showing a prolonged negative response. Ludvigson, $\mathrm{Ma}$ and $\mathrm{Ng}$ (2015) address the question on whether uncertainty and real economic activity could affect one another contemporaneously. They find that higher uncertainty in recessions is endogenous to business cycle fluctuations.

Here we compare the effects of macroeconomic and financial uncertainty, on macroeconomic variables, by extending the analysis in previous literature to a panel of countries, allowing for the possibility of switching in the effects of the two types of uncertainty over the business cycle, and to data at different frequencies. We are mainly interested in studying the asymmetric effects in the impact and delayed effects of uncertainty on each variable, where uncertainty is measured at a frequency higher than the macroeconomic variables. Thus, we make the simplifying assumption that uncertainty is exogenous, while in Jurado, Ludvigson and $\mathrm{Ng}$ (2015) and Carriero, Clark and Marcellino (2018) there is a feedback from the macroeconomic variables to (low frequency) uncertainty. This is relevant to study the dynamic propagation of uncertainty shocks in the economic system.

3.2. Dataset. We consider a panel of $G=13$ countries: United States (US), Europe (EU), Japan (JP), Germany (DE), France (FR), United Kingdom (UK), Italy (IT), Canada (CA), the Netherlands (NE), Norway (NW), Spain (SP), Sweden $(\mathrm{SW})$ and Switzerland $(\mathrm{CH})$. The choice of the countries is based on the availability of data from the Consensus Economics, from which we construct our measure of macroeconomic uncertainty.

Our macroeconomic uncertainty measure is based on the disagreement about the projections for the real GDP growth among the professional forecasters participating to the Consensus economic polls. Our uncertainty measure consists of the standard deviations of the said projections. A clarification on how to compute the measure is needed. The respondents to the survey are asked to give their expectations on the current and next calendar year. Following Dovern, Fritsche and Slacalek (2012) and Ferrara and Guerin (2015), we construct our measure of disagreement on one-year-ahead forecasts. Thus, we first transform fixed-event forecasts into fixed-horizon forecasts, by taking the average of the forecasts for the current and next calendar year weighted by their share in the forecasting horizon:

$$
x_{t}^{e}=\frac{\mathcal{K}}{12} x_{t+\mathcal{K} \mid t}+\frac{12-\mathcal{K}}{12} x_{t+12+\mathcal{K} \mid t} .
$$


Here $x_{t}^{e}$ is the one-year-ahead expectation, $x_{t+\mathcal{K} \mid t}$ is the current-year expectation, $x_{t+12+\mathcal{K} \mid t}$ is the next-year expectation and $\mathcal{K}$ are the months to the end of the current year at the moment the survey is made. The standard deviation is then computed on this one-year-ahead expectation.

As a measure of financial uncertainty we consider the US VIX, as in Bloom (2009). We consider the US measure also when looking at the uncertainty in other countries because it is the longest series available. Further, we computed the correlation of the US VIX with the series of VIX available for other countries (e.g. UK, Canada, Italy) and the correlation is high. Figure D.4 in Supplement D shows the three-year rolling correlation of the US VIX with the VIX for the other countries we have available. In Figure D.5 we show the correlation of the VIX with the macroeconomic uncertainty measures for each country. The three monthly series are kept separately in this graph. In Supplement D, we repeat the exercise using the Financial Uncertainty Index developed in Ludvigson, $\mathrm{Ma}$ and $\mathrm{Ng}$ (2015) for the US economy and a financial uncertainty measure based on systemic risk and proposed in Billio et al. (2016a) for the EU financial market.

The macroeconomic variables we consider for each country in the panel are the following: real GDP (labeled GDP in tables) and industrial production growth rates (IPI), employment growth rate (Emp), consumption growth (Con), inflation (Inf), nominal earnings growth (Ner), real earnings growth (Rer), working hours growth (Hours), nominal interest rates (IR), stock market index growth (Stock) and monetary M2 aggregate growth (M2). All variables are sampled at a quarterly frequency from 1997 to 2014. The availability of the data for each country, the sample period and the source are given in Supplement D. Figures D.1-D.2 show the dependent variables in the panel. Figure D.3 shows the exogenous variables (i.e., forecast disagreement and VIX). All the variables are standardized to have comparable scales for the coefficients. See figures E.3-E.5 in Supplement E for the impact of uncertainty on the different variables in the original variable-specific scale.

The uncertainty variables are collected at monthly frequency and the other macroeconomic variables at quarterly frequency. The model uses four lags for the quarterly variables, two lags (contemporaneous and 1-quarter lag) for the monthly variables, and two regimes. The first regime requires that the common intercepts $\boldsymbol{\mu}$ are nonpositive. We define it as contraction regime. In the second regime, the common intercepts are equal to zero or positive. We define it as expansion regime. Economic theory supports that the variable M2 can both grow or decrease in contraction periods. We notice that in all the countries in our dataset M2 mostly increases during recessions, therefore we take minus its growth rate and apply the described restriction. As mentioned, the use of different regimes and of data at different frequencies attenuates possible endogeneity problems of uncertainty.

3.3. Results. We first look at the country- and series-specific impact $\delta_{i j d g k}=$ $\delta_{j d k}+\zeta_{\delta, g j d k}+\eta_{\delta, i g j d k}$ in the two regimes, where $i$ refers to the macroeconomic variable, $j$ indicates the covariate, and we define $j=1$ the macroeconomic uncertainty and $j=2$ the financial uncertainty; $d$ indicates the lag. We 
consider six months of past information and correspondingly six coefficients (i.e., $d=0, \ldots, 5)$, where $g=1, \ldots, 13$ indicates the country, and $k$ indicates the regime, with $k=1,2$ referring respectively to contraction and expansion.

In Table 1 we provide a summary representation of the coefficients by reporting the median of the sum of the six coefficients for the monthly contemporaneous and lagged variables, that have $90 \%$ of the mass different from zero for the two regimes. This allows us to take into account the parameter uncertainty in the results, since we sum the full distributions of the coefficients and we drop those that include zero in the $90 \%$ highest posterior density (HDP). Table 1 reports results only for the VIX because the HDP of coefficients associated to macroeconomic uncertainty includes zero in all cases, confirming the marginal role of macroeconomic uncertainty with respect to financial uncertainty. In all cases, the sum of coefficients is larger in absolute value in regime 1 than in regime 2, supporting evidence in Caggiano, Castelnuovo and Groshenny (2014), Alessandri and Mumtaz (2014) and Ludvigson, Ma and $\mathrm{Ng}$ (2015) that uncertainty has a bigger effect in recessions (regime 1). Moreover, the sum of coefficients is significant for both regimes for almost all countries for the variables GDP, Industrial production, real earnings, stock markets, M2, whereas it sometimes includes zero in the HDP for employment, consumption, inflation, nominal earnings and hours, in particular for the expansionary regime. Further, the effect of financial uncertainty on interest rate is homogeneous across 10 countries, but it differs for Japan (not significant on both regimes), Canada and Switzerland (not significant in expansion). The Abenomics for Japan, the stronger expansion of Canada in periods of high US financial uncertainty, and the central bank interventions on the Swiss exchange rate are possible explanations for this finding.

We then move to look at the disaggregated results in more detail. Figures 1 to 3 show the impact of uncertainty on the variables of different countries for the two regimes (contraction and expansion), at different lags. In each figure, the left column plots the impact of the forecast disagreement $(j=1)$ and the right column plots the impact of VIX $(j=2)$. The regimes extracted for each country are reported in Figures E.1-E.2 in Supplement E.

Each plot of the Figures 1 to 3 reports the results for a variable $i$. The circles represent the common impact in each of the two regimes, that is they represent the pair $\left(\delta_{j d 1}, \delta_{j d 2}\right)$, with $\delta_{j d k}, k=1,2$ defined as in equation (2.6). In the figures the circles are indicated with the pair $(j, d)$, referring to the pair of covariate $(j=1,2)$ and lag $(d=0, \ldots, 5)$. Notice that the circles are the same across plots for every variable $i$, but they differ between the plots in the first and second column (given the different covariate indexed by $j$ ). The dots instead represent country- and series-specific impact in the two regimes, that is, they represent the pair $\left(\delta_{i j d g 1}, \delta_{i j d g 2}\right)$ for all countries, where $\delta_{i j d g k}$ is defined as in equation (2.6). The dots are different in each plot, given that they are different for each variable.

The first clear result is that for all variables, in all countries and both measures of uncertainty, all the estimates (intended as the mean of the posterior distributions) 
TABLE 1

Effects of financial uncertainty (sum of the VIX coefficients) on the different variables in the 13 countries, in regime 1 (recession, first column) and regime 2 (expansion, second column). Symbol “-”: not available. Empty cell: not significantly different from zero

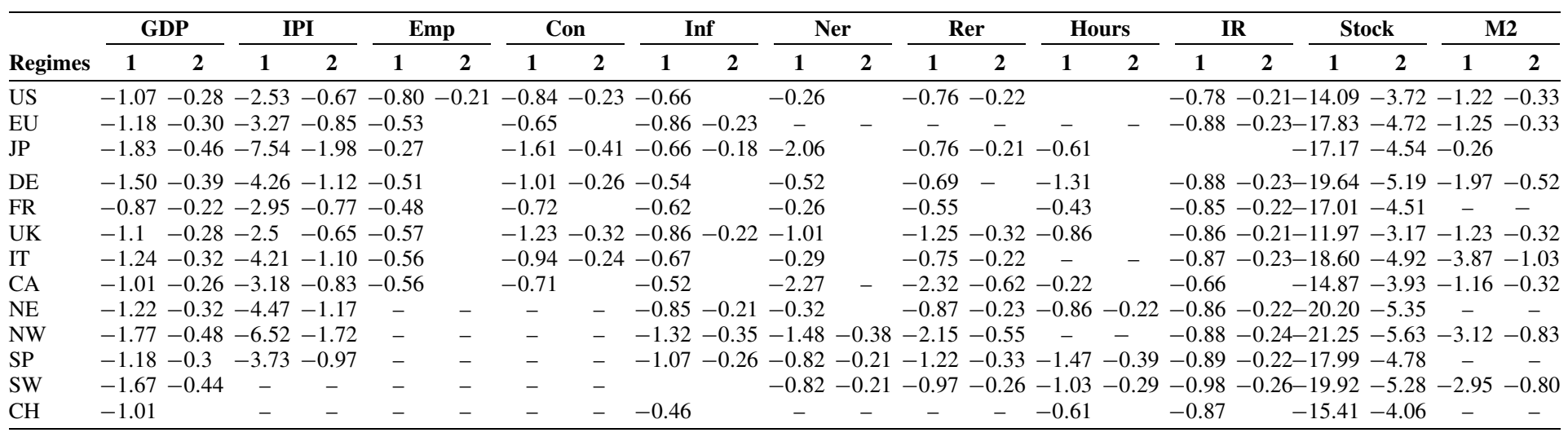


GDP-Dis

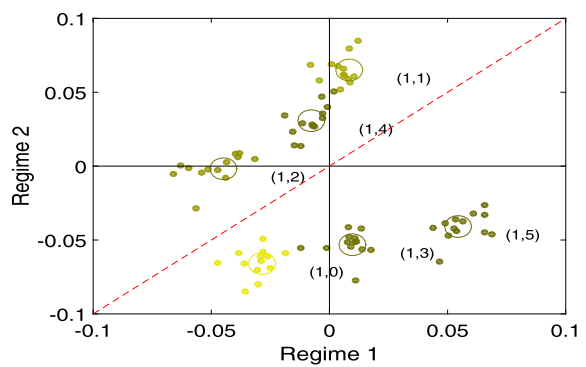

IPI-Dis

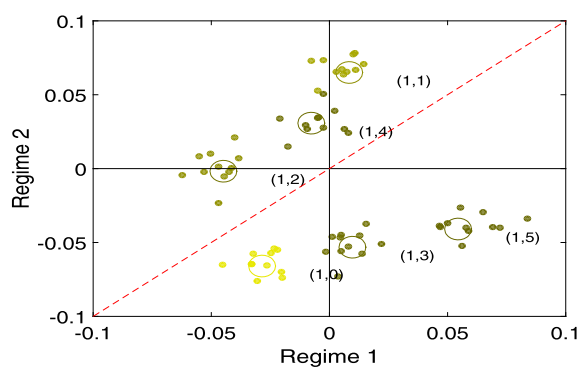

Employment-Dis

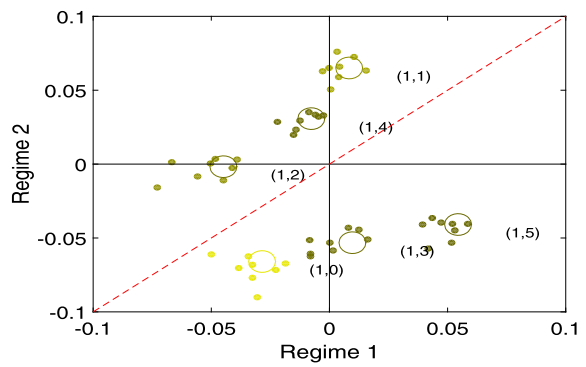

Consumption-Dis

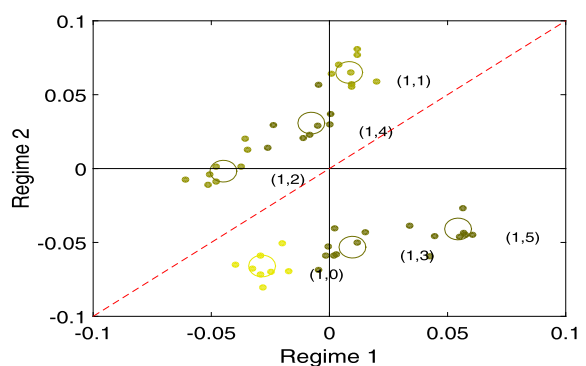

GDP-VIX

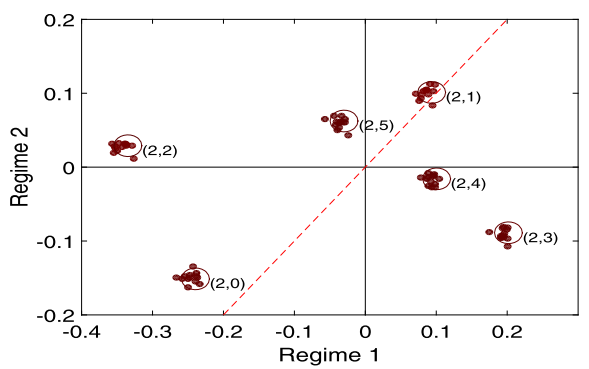

IPI-VIX

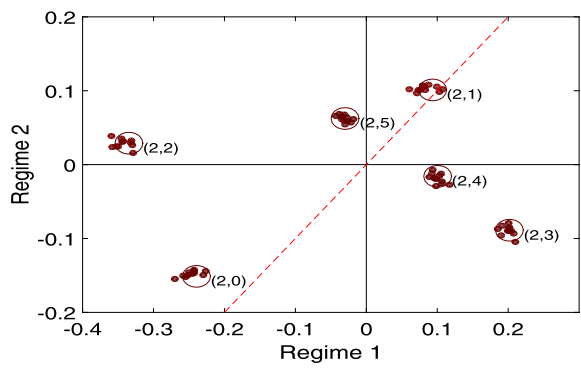

Employment-VIX

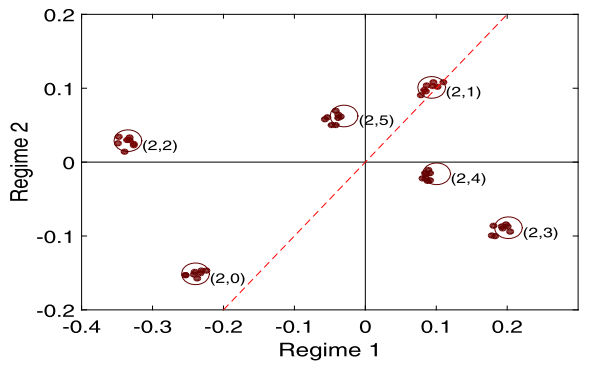

Consumption-VIX

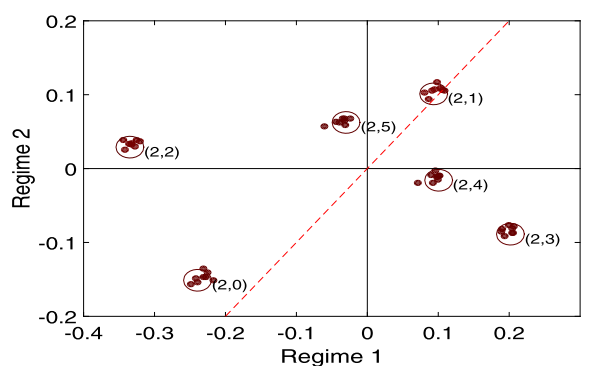

FIG. 1. Impact of uncertainty on different macroeconomic variables. Different plots: impact of macroeconomic (left) and financial (right) uncertainty on each variable $i=1, \ldots, n_{g}$ for all the countries $g=1, \ldots, G$, in the two regimes $k=1,2$ (respectively, contraction and expansion). Circles: common impact $\delta_{j d k}$, where $j=1,2$ refers to forecast disagreement and VIX, respectively, and $d=0, \ldots, 5$ to the lag. Dots: country-and series-specific impact $\delta_{i j d g k}$ as in equation (2.6), in the two regimes. Shades of color: refer different pairs $(j, d)$. Dashed line: the $45^{\circ}$ line. 
Nominal ER-Dis

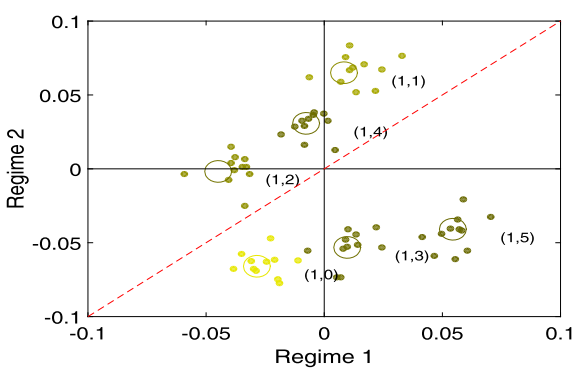

Real ER-Dis

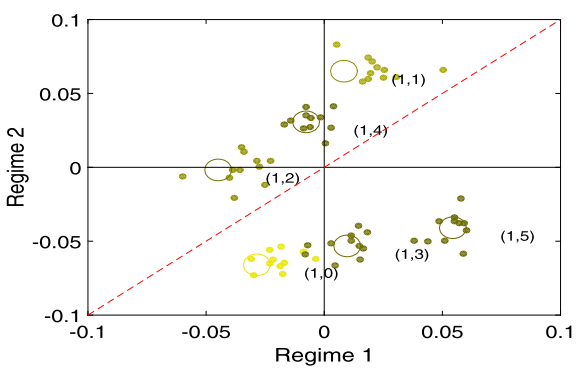

Inflation-Dis

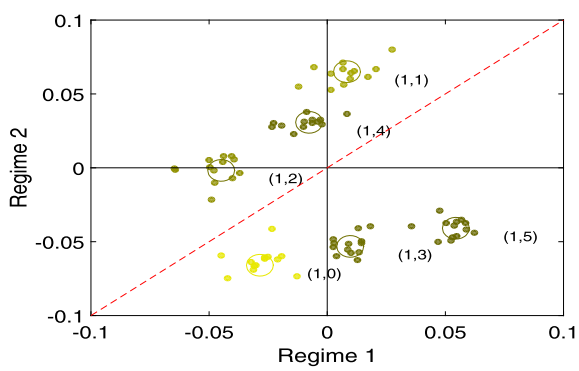

Hours-Dis

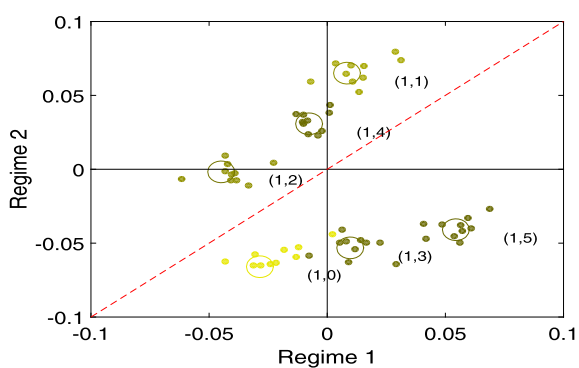

Nominal ER-VIX

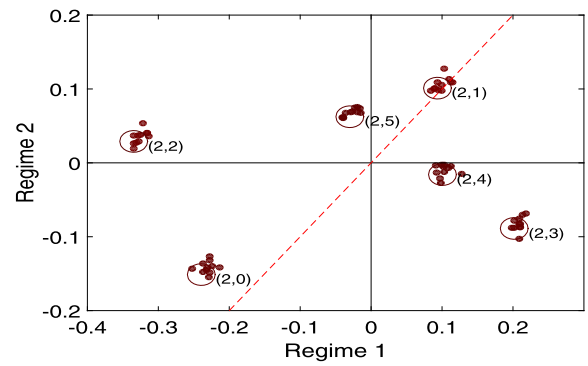

Real ER-VIX

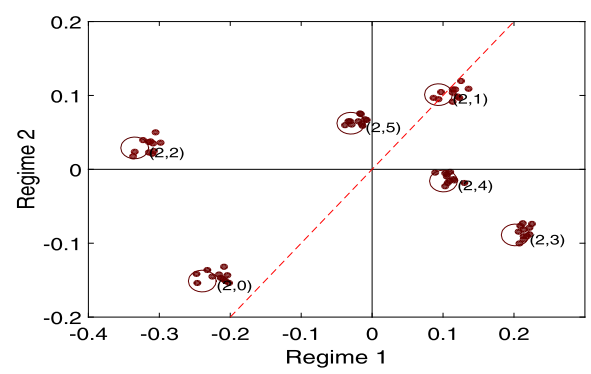

Inflation-VIX

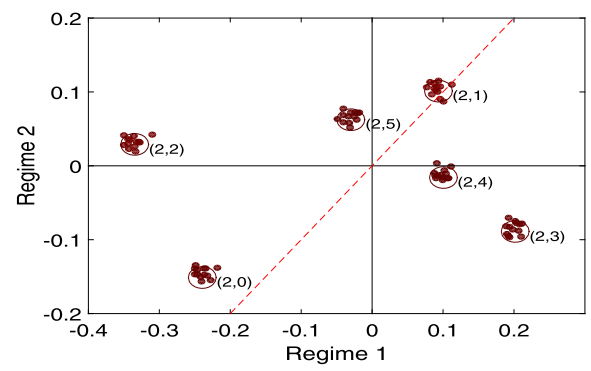

Hours-VIX

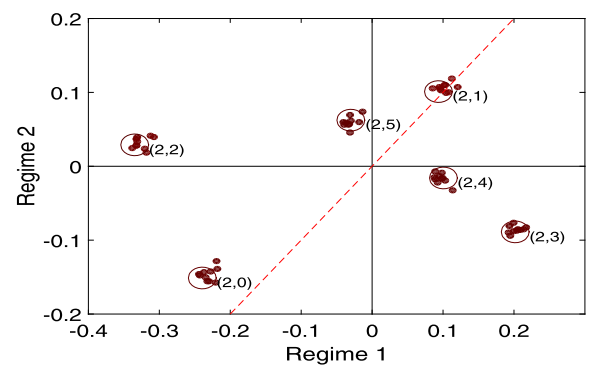

FIG. 2. Impact of uncertainty on different macroeconomic variables. Different plots: impact of forecast disagreemnt (left) and VIX (right) on each variable $i=1, \ldots, n_{g}$ for all the countries $g=1, \ldots, G$, in the two regimes $k=1,2$ (respectively, contraction and expansion). Circles: common impact $\delta_{j d k}$, where $j=1,2$ refers to forecast disagreement and VIX, respectively, and $d=0, \ldots, 5$ to the lag. Dots: country- and series-specific impact $\delta_{i j d g k}$ as in equation (2.6), in the two regimes. Shades of color: refer different pairs $(j, d)$. Dashed line: the $45^{\circ}$ line. 
IR-Dis

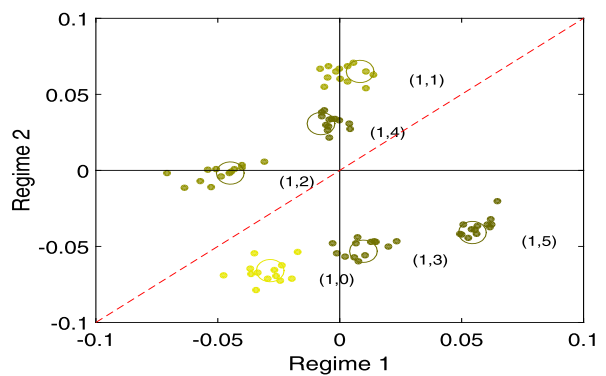

Stock-Dis

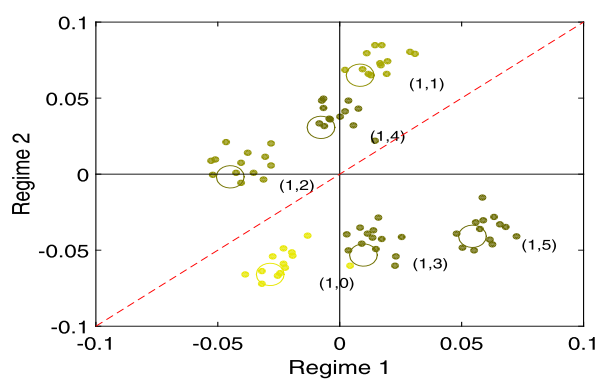

M2-Dis

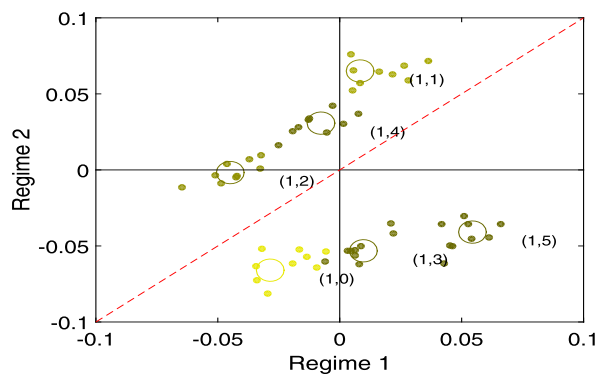

IR-VIX

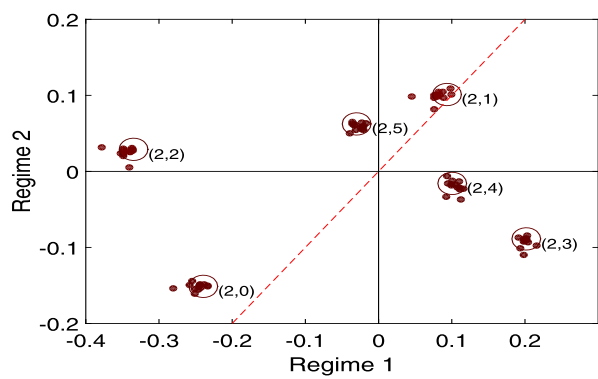

Stock-VIX

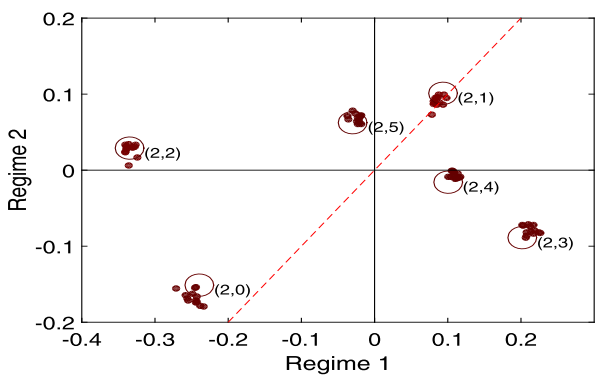

M2-VIX

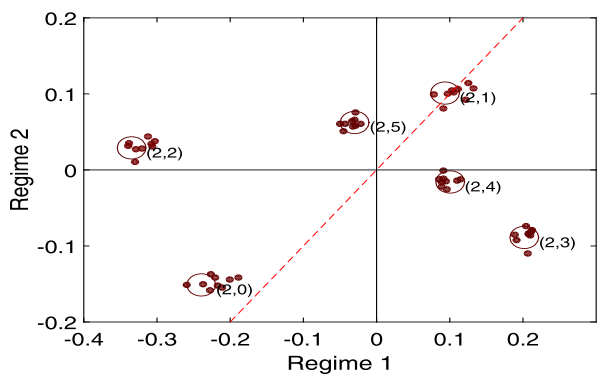

FIG. 3. Impact of uncertainty on different macroeconomic variables. Different plots: impact of forecast disagreement (left) and VIX (right) on each variable $i=1, \ldots, n_{g}$ for all the countries $g=1, \ldots, G$, in the two regimes $k=1,2$ (respectively, contraction and expansion). Circles: common impact $\delta_{j d k}$, where $j=1,2$ refers to forecast disagreement and VIX, respectively, and $d=0, \ldots, 5$ to the lag. Dots: country- and series-specific impact $\delta_{i j d g k}$ as in equation (2.6), in the two regimes. Shades of color: refer different pairs $(j, d)$. Dashed line: the $45^{\circ}$ line.

are not on the 45 degree line. This means that there is an asymmetric effect of the uncertainty across regimes. For the macroeconomic uncertainty, measured as forecast disagreement, in the contraction regime, contemporaneous shocks in the first and third months [labelled $(1,0)$ and $(1,2)]$ have negative impact on GDP, IPI and consumption, whereas at one quarter lag [labelled $(1,3)$ and $(1,5)]$ have positive impact on GDP, IPI and consumption. This seems to confirm the drop, rebound and 
overshoot dynamics described in Bloom (2009). For the same variables, in the expansion regime the coefficients $(1,0),(1,3)$ and $(1,5)$ are negative whereas $(1,1)$, and $(1,4)$ are positive. So, the effects are different in the two regimes, confirming the asymmetric evidence in Caggiano, Castelnuovo and Groshenny (2014).

The same results apply to the VIX: in the contraction regime the effects in first and third month [labeled by $(2,0)$ and $(2,2)$ in the plots] are negative on GDP, IPI and consumption, whereas at one quarter lag the first and second months [labelled $(2,3)$ and $(2,4)]$ have a positive impact on GDP, IPI and consumption. In the expansion regime, the coefficients $(2,0),(2,3)$ and $(2,4)$ are negative whereas $(2,1),(2,2)$ and $(2,5)$ are positive.

The outcome is similar for other macroeconomic variables, even if some of the coefficients are closer to zero, in particular those indicating the effect of macroeconomic uncertainty. For example, the coefficients for forecast disagreement of the third month in the first regime $(1,2)$ for employment, nominal and real exchange rates, inflation, hours, interest rate, stock returns and M2 are basically zero for all countries. Such pattern is less evident for the VIX coefficients where only in few occasions the coefficient is zero.

The second important finding is that the coefficients of financial uncertainty are in almost all cases larger than those of macroeconomic uncertainty. For example, a $1 \%$ increase in financial uncertainty in the first month of the quarter results in the first regime in a reduction of quarterly GDP of at least $0.2 \%$ for all countries, and in the second regime in a reduction of quarterly GDP around $0.15 \%$ for all countries. Similar evidence is found for other variables, confirming the evidence in Caggiano, Castelnuovo and Groshenny (2014), who focus only on unemployment.

On the contrary, a $1 \%$ increase in macroeconomic uncertainty in the first month of the quarter results in a reduction of GDP around $0.05 \%$ in recession, and in a reduction of GDP bigger than $0.05 \%$ in expansion. Similar evidence is found for the other variables. Interestingly, financial uncertainty shocks cause larger drops in the contraction regime, whereas macroeconomic uncertainty shocks in the expansion regime. Our measure of financial uncertainty, the VIX, incorporates a risk premium component, that is not necessarily included in the macroeconomic measure, and this could possibly explain the stronger link between it and our macroeconomic variables.

The plots show that the effects of the VIX are more homogeneous across countries, with most of the coefficients close to the common impacts; on the contrary, more heterogeneity exists for the forecast disagreement. The result can be explained by the fact that we use the same US VIX variable for all countries, as it is highly correlated to the VIX of the countries for which it is available, even if for a shorter sample. Forecast disagreement is instead different across countries, suggesting that financial uncertainty shocks are rather uniform across the countries in our sample, whereas macroeconomic uncertainty shocks depend more on domestic economic conditions. 


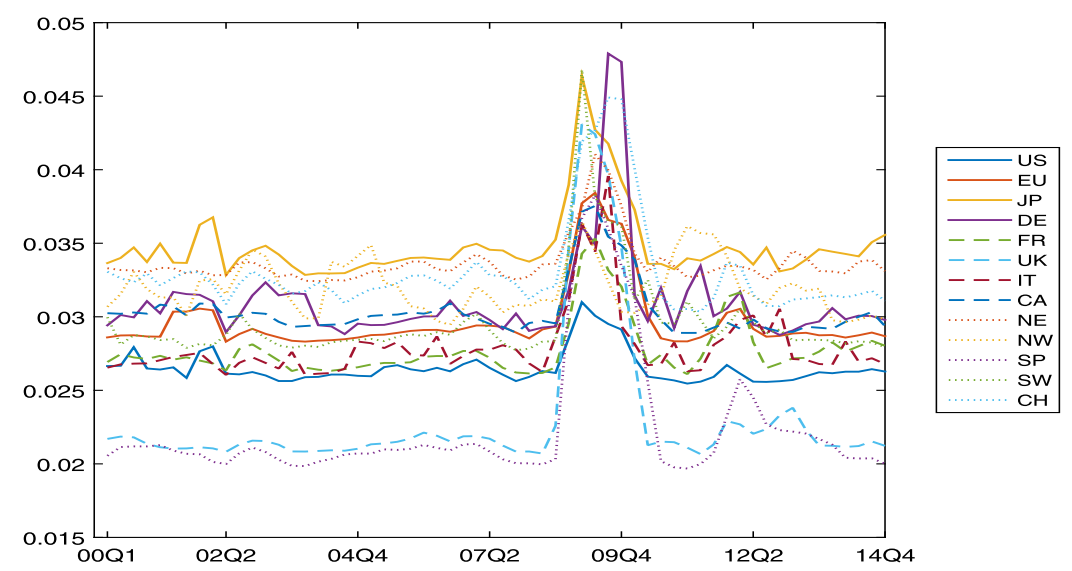

FIG. 4. Estimated time-varying volatility $\hat{\sigma}_{\text {igt }}$ for the GDP of different countries (coloured lines).

We find evidence of heteroschedasticity in all variables and countries. Figure 4 shows the estimated time-varying volatility for the GDP of the different countries averaged over different regimes. For the plot of the raw series of GDP see the top-left panel in Figure D.1 in Supplement D.

$$
\hat{\sigma}_{i g t}^{2}=\sum_{k=1}^{K}\left\{\hat{\sigma}^{2} \hat{\lambda}_{i g}^{-1} \hat{\chi}_{g}^{-1}+\hat{\mathbf{z}}_{i g t}^{\prime}\left(\hat{R}+\hat{Q}_{g}\right) \hat{\mathbf{z}}_{i g t}\right\} p_{g k t},
$$

where $\mathbf{z}_{i g t, k}^{\prime}=\left(\mathbf{e}_{k}^{\prime}, \mathbf{v}_{i g t}^{\prime} \otimes \mathbf{e}^{\prime}, \mathbf{x}_{i g, 1 t}^{\prime} \otimes \mathbf{e}^{\prime}, \ldots, \mathbf{x}_{i g, N t} \otimes \mathbf{e}_{k}^{\prime}\right)$ is the time-varying volatility at time $t$ conditional to regime $k, \mathbf{e}_{k}$ is the $k$ th vector of the standard orthonormal basis, $p_{g k t}$ is the posterior probability for the country $g$ of being in regime $k$, that is, $(\operatorname{Pr})\left(\boldsymbol{\xi}_{g t}=\mathbf{e}_{k} \mid \mathbf{y}\right)$, and $\hat{\boldsymbol{\theta}}$ is the posterior mean of $\boldsymbol{\theta}$. The error volatility increases substantially during the 2008-2009 financial crisis, as documented in other studies [Clark and Ravazzolo (2015)] and returns to precrisis level for several countries after 2010. There are, however, important differences across countries. Non-euro countries, such as Japan, Sweden, UK and the US, seem to lead the increase from the end of 2007 to 2008, whereas euro country estimates had the highest values in 2009; see, for example, Germany. This confirms evidence that the US cycle often leads euro country cycles; see Billio et al. (2016b). Moreover, euro-country estimates remain more volatile after 2010, in conjunction with the intensification of the European debt crisis.

3.4. Comparison with constrained models. As mentioned in Section 2, our model extends the work of Kaufmann (2010, 2015) by allowing for mixedfrequencies. In this section we then compare our results to those which can be obtained by shutting off the mixed-frequency component (i.e., we restrict the model coefficients to be the same for the months within a quarter). Further, as a second 

GDP-Dis

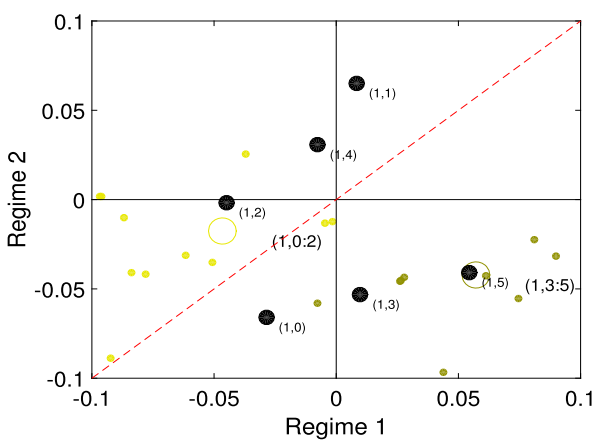

Panel (a): no mixed-frequency mechanism

GDP-VIX

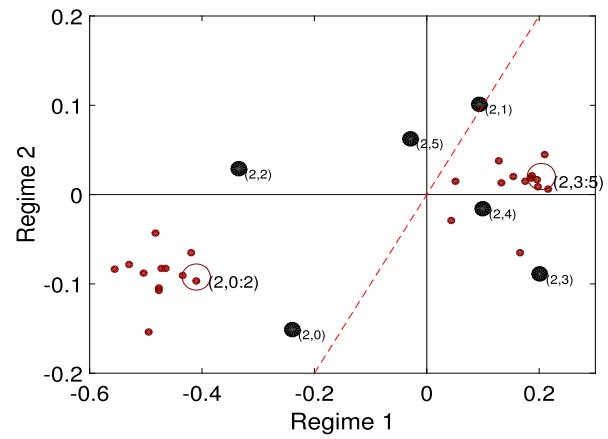

Panel (b) no regime switching mechanism

GDP-Dis

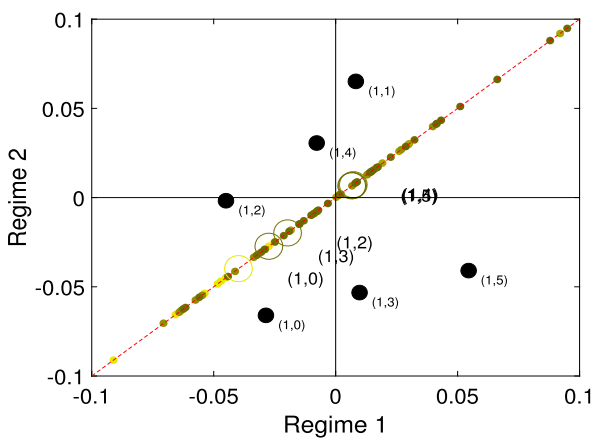

GDP-VIX

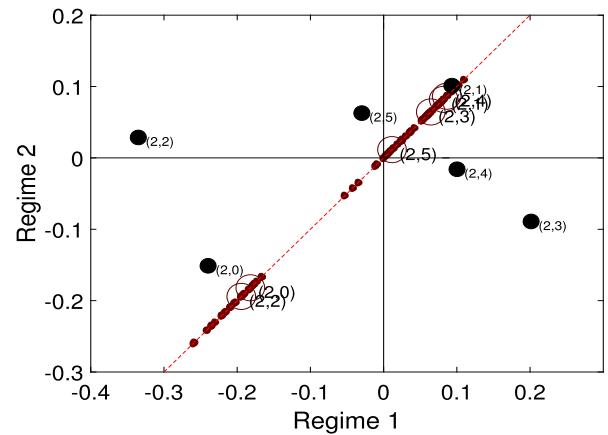

FIG. 5. Impact of uncertainty in constrained models. Different plots: impact of forecast disagreement (left) and VIX (right) on GDP for all the countries $g=1, \ldots, G$, in the two regimes $k=1,2$ (respectively, contraction and expansion) for two constrained models: model without mixed frequency effects [Panel (a)] and model without regime-switching effects [Panel (b)]. Circles: common impact $\delta_{j d k}$, where $j=1,2$ refers to forecast disagreement and VIX, respectively, and $d=0, \ldots, 5$ to the lag. Dots: country- and series- specific impact $\delta_{i j d g k}$ as in Eq. (2.6), in the two regimes. Shades of color: refer different pairs $(j, d)$. Dashed line: the $45^{\circ}$ line. Black dots: the common factors estimates for the unconstrained model reported in Figure 1.

comparison, we remove the Markov-switching mechanism and see how a linear model would fit our experiment.

The results are shown in Figure 5. For the sake of conciseness, we focus on the impact of forecast disagreement and VIX on GDP only for the different countries. In Panel (a), we report the results for the case in which the mixed-frequency component is disregarded, while in Panel (b) we report the results without regime switching.

Interestingly, when ignoring the mixed frequency component, the effects of uncertainty appear different. In particular, opposite to the results for the unconstrained MIDAS model (indicated by the black dots in the plot), the effects of 
VIX have the same sign across regimes [see dots and circles labelled $(2,0: 2)$ and $(2,3: 5)]$ : negative for the contemporaneous quarter and positive for the one-lag quarter. For the forecast disagreement, the use of quarterly uncertainty seems to capture only what happens in the third month of the quarter [with $(1,0: 2)$ similar to $(1,2)$ and $(1,3: 5)$ to $(2,5)]$.

When ignoring the regime switching mechanism, we also weaken our results, especially in the case of the VIX. Results become more similar to the ones obtained in the contraction regime when the switching is allowed, losing the asymmetric effect in expansions. For the VIX case, for example, the effect is negative in the first and third month of the contemporaneous quarter; see circles $(2,0),(2,2)$, and positive otherwise.

3.5. Further robustness. We summarize here the results of a set of additional robustness checks that we conducted in order to strengthen our results. The full set of results is shown in Supplement E. First, we remove the contemporaneous effect of financial and macroeconomic uncertainty and only consider the three months of the one-quarter lag. This choice shall remove any possible remaining effects of endogeneity of the uncertainty variables that the Markov-switching mixed frequency approach cannot capture. Second, we include in our panel MIDAS model only the VIX. Third, we include only the forecast disagreement. For both the second and third cases, we study a model with uncertainty used at higher frequency, but also as quarterly average, to provide further evidence on the usefulness of mixed frequency data. Fourth, we investigate different measures of financial uncertainty, addressing the concern that VIX might not be the best measure to account for such uncertainty; see, for example, Jurado, Ludvigson and $\mathrm{Ng}$ (2015). We consider the Financial Uncertainty Index developed in Ludvigson, Ma and $\mathrm{Ng}$ (2015) and the Financial Entropy Index proposed in Billio et al. (2016a). The former index is built on a large set of financial variables using a new methodology called iterative projection IV. The indicator is only available for the US. The latter index captures the level of systemic risk and measures the entropy of the loss cascades on the financial market. It detects loss cascades by $\triangle \mathrm{CoVaR}$, which represents the value at risk (VaR) of the financial system conditional on institutions being under distress. Therefore, such measure amplifies the risk premium component of financial uncertainty by using a systemic risk early warning. The entropy indicator for the EU is built on a dataset of daily closing price series for the European firms (active and dead) of the financial sector from January 1990 to December 2014. In both cases, we follow the same assumption applied to the VIX and use it for all countries.

First, the evidence is qualitatively similar when the model only allows for a onequarter lag effect of uncertainty. Financial uncertainty still dominates on macroeconomic uncertainty and differences across regimes clearly emerge.

When dropping the VIX from the model, coefficients of forecast disagreement become larger in absolute value, probably capturing part of the effects that our 
model assigns to financial uncertainty. When we use quarterly uncertainty measures, the heterogeneity across countries for both VIX and forecast disagreement increases substantially, and for several variables the total effect of uncertainty is less pronounced, especially for the second regime, confirming the usefulness of mixed frequency data.

The main results are also confirmed when using different measures of financial uncertainty. Both the Financial Uncertainty Index of Ludvigson, $\mathrm{Ma}$ and $\mathrm{Ng}$ (2015) and the Financial Entropy Index of Billio et al. (2016a) yield a negative impact (mainly) during the recession regime, thus supporting evidence of asymmetric effects of uncertainty in different phases of the economic activity.

4. Conclusions. This paper develops a Bayesian multi-country panel Markovswitching unrestricted mixed-data sampling model. This framework allows to model a large panel of countries and several variables for each country, to use an endogenous time-varying transition mechanism, to model nonlinearities, and to consider variables sampled at mixed frequencies. To avoid overparameterization issues and overfitting problems, we implement a hierarchical strategy in the specification of the prior. The hierarchical prior allows to naturally introduce random effects into the panel model without specifying a Markov-switching mechanism in the variance of the errors. We develop an MCMC algorithm for sampling from the joint posterior distribution of the model parameters and test its properties in a simulation experiment.

We use the model to study the effects of macroeconomic uncertainty, measured as forecast disagreement, and financial uncertainty, measured as stock market volatility, on a set of variables in a multi-country context including the US, several European countries and Japan. We find that for most of the variables financial uncertainty dominates macroeconomic uncertainty. Furthermore, we show that uncertainty coefficients differ if the economy is in a contraction regime or in an expansion regime.

\section{APPENDIX A: PROOFS}

PROPOSITION 1. The model in equation (2.7) can be written as:

$$
y_{i g t}=\mathbf{z}_{i g t}^{\prime}\left(\boldsymbol{\beta}+\zeta_{g}+\eta_{i g}\right)+\varepsilon_{i g t},
$$

with $\boldsymbol{\beta}=\left(\boldsymbol{\mu}, \mathbf{c}_{1}, \ldots, \mathbf{c}_{c}, \boldsymbol{\delta}_{10}, \ldots, \boldsymbol{\delta}_{N v}\right)^{\prime}$ the parameter vector, $\mathbf{z}_{i g t}=\left(\boldsymbol{\xi}_{g t}^{\prime}\right.$, $\left.\mathbf{v}_{i g t}^{\prime} \otimes \xi_{g t}^{\prime}, \mathbf{x}_{i g, 1 t}^{\prime} \otimes \xi_{g t}^{\prime}, \ldots, \mathbf{x}_{i g, N t}^{\prime} \otimes \xi_{g t}^{\prime}\right)^{\prime}$ the covariate vector of dimension $K(1+c+N(v+1)) \times 1, \boldsymbol{\eta}_{i g}=\left(\boldsymbol{\eta}_{\mu, i g}, \boldsymbol{\eta}_{c, i g 1}, \ldots, \boldsymbol{\eta}_{c, i g c}, \boldsymbol{\eta}_{\delta, i g 10}, \ldots, \boldsymbol{\eta}_{\delta, i g N v}\right)^{\prime}$ the variable-specific random effects vector, and $\zeta_{g}=\left(\zeta_{\mu g}, \zeta_{c, g 1}, \ldots, \zeta_{c, g c}\right.$, $\left.\zeta_{\delta, g 10}, \ldots, \zeta_{\delta, g N v}\right)^{\prime}$ the country-specific random effects vector.

PROOF. Let $C=\left(\boldsymbol{c}_{1}, \ldots, \boldsymbol{c}_{c}\right), Z_{c, g}=\left(\boldsymbol{\zeta}_{c, g 1}, \ldots, \boldsymbol{\zeta}_{c, g c}\right), E_{c, i g}=\left(\boldsymbol{\eta}_{c, i g 1}, \ldots\right.$, $\left.\boldsymbol{\eta}_{c, i g c}\right)$ three $K \times c$-matrices, and $D_{j}=\left(\boldsymbol{\delta}_{j 1}, \ldots, \boldsymbol{\delta}_{j(v+1)}\right), Z_{d, g j}=\left(\boldsymbol{\zeta}_{\delta, g j 1}, \ldots\right.$, 
$\left.\zeta_{\delta, g j v+1}\right), E_{d, i g}=\left(\boldsymbol{\eta}_{\delta, i g j 1}, \ldots, \boldsymbol{\eta}_{\delta, i g j v+1}\right)$ three $K \times(v+1)$-dimensional matrices, then equation (2.7) can be rewritten as

$$
\begin{aligned}
y_{i g t}= & \left(\boldsymbol{\mu}+\zeta_{\mu, g}+\boldsymbol{\eta}_{\mu, i g}\right)^{\prime} \boldsymbol{\xi}_{g t}+\mathbf{v}_{i g t}^{\prime}\left(C+Z_{c, g}+E_{c, i g}\right)^{\prime} \boldsymbol{\xi}_{g t} \\
& +\sum_{j=1}^{N} \mathbf{x}_{i g, j t}^{\prime}\left(D_{j}+Z_{d, g j}+E_{d, i g j}\right)^{\prime} \boldsymbol{\xi}_{g t}+\varepsilon_{i g t},
\end{aligned}
$$

with $\varepsilon_{i g t} \sim \mathcal{N}\left(0, \sigma_{i g}^{2}\right)$, where $\sigma_{i g}^{2}$ is defined in equation (2.6).

Let $\mathbf{w}_{i t}=\left(1, \mathbf{v}_{i g t}^{\prime}, \mathbf{x}_{i g t}^{\prime}\right)^{\prime}$, where $\mathbf{x}_{i g t}=\left(\mathbf{x}_{i g, 1 t}^{\prime}, \ldots, \mathbf{x}_{i g, N t}^{\prime}\right)^{\prime}$, and the $K \times(1+$ $c+N(v+1))$-matrices $B=\left(\boldsymbol{\mu}, C, D_{1}, \ldots, D_{N}\right), Z_{g}=\left(\zeta_{\mu, g}, Z_{c, g}, Z_{d, g}, \ldots\right.$, $\left.Z_{d, g N}\right)$ and $E_{i g}=\left(\eta_{\mu, i g}, E_{c, i g} E_{d, i g 1}, \ldots, E_{d, i g N}\right)$. Then equation (A.2) becomes:

$$
y_{i g t}=\mathbf{w}_{i t}^{\prime}\left(B+Z_{g}+E_{i g}\right)^{\prime} \boldsymbol{\xi}_{g t}+\varepsilon_{i g t} .
$$

Applying the vec operator, its properties and the properties of the Kronecker's product, $\otimes$, [Magnus and Neudecker (1999), pages 31-32] we obtain:

$$
\begin{aligned}
\operatorname{vec}\left(y_{i g t}\right) & =\operatorname{vec}\left(\boldsymbol{\xi}_{g t}^{\prime}\left(B+Z_{g}+E_{i g}\right) \mathbf{w}_{i t}+\varepsilon_{i g t}\right) \\
& =\left(\mathbf{w}_{i t}^{\prime} \otimes \boldsymbol{\xi}_{g t}^{\prime}\right) \operatorname{vec}\left(\left(B+Z_{g}+E_{i g}\right)\right)+\varepsilon_{i g t} \\
& =\mathbf{z}_{i t}^{\prime}\left(\boldsymbol{\beta}+\zeta_{g}+\eta_{i g}\right)+\varepsilon_{i g t},
\end{aligned}
$$

where $\mathbf{z}_{i t}^{\prime}=\mathbf{w}_{i t}^{\prime} \otimes \xi_{i t}^{\prime}=\left(\xi_{i t}^{\prime}, \mathbf{v}_{i g t}^{\prime} \otimes \xi_{i t}^{\prime}, \mathbf{x}_{i g t}^{\prime} \otimes \xi_{i t}^{\prime}\right), \boldsymbol{\beta}=\operatorname{vec}\left((B), \zeta_{g}=\operatorname{vec}\left(Z_{g}\right)\right.$ and $\boldsymbol{\eta}_{i g}=\operatorname{vec}\left(E_{i g}\right)$.

PROPOSITION 2. Marginalizing out the random effects in the right-hand side of equation (A.1) one obtains

$$
y_{i g t}=\mathbf{z}_{i g t}^{\prime} \boldsymbol{\beta}+\tilde{\varepsilon}_{i g t}, \quad \tilde{\varepsilon}_{i g t} \sim \mathcal{N}\left(0, \sigma_{i g t}^{2}\right),
$$

for $t=m(c+1), 2 m, \ldots, m T_{q}$, with $\sigma_{i g t}^{2}=\sigma^{2} \lambda_{i g}^{-1} \chi_{g}^{-1}+\mathbf{z}_{i g t}^{\prime}\left(R+Q_{g}\right) \mathbf{z}_{i g t}$.

ProOF. From Proposition 1 it follows that $\boldsymbol{\beta} \sim \mathcal{N}_{d}\left(\mathbf{0}_{d}, S\right)$ with $d=K(1+$ $c+N(v+1))$ and $S=\operatorname{diag}\left\{\left(s_{0}^{2} \iota_{K}^{\prime}, r_{0}^{2} \iota_{K(c+N(v+1))}^{\prime}\right\}\right.$ a diagonal covariance matrix and $\iota_{q}$ the $q$-dimensional country vector. Also, $\zeta_{g} \sim \mathcal{N}_{d}\left(\mathbf{0}_{d}, R\right)$ with $R=\operatorname{diag}\left\{\left(\left(r_{\mu, 1}, \ldots, r_{\mu, K}\right), \iota_{c}^{\prime} \otimes\left(r_{c, 1}, \ldots, r_{c, K}\right), \iota_{N(v+1)}^{\prime} \otimes\left(r_{\delta, 1}, \ldots, r_{\delta, K}\right)\right)^{\prime}\right\}$, and $\eta_{i g} \sim \mathcal{N}_{d}\left(\mathbf{0}_{d}, Q_{g}\right)$ where $Q_{g}=\operatorname{diag}\left\{\left(\left(q_{\mu, g 1}, \ldots, q_{\mu, g K}\right), \boldsymbol{\iota}_{c}^{\prime} \otimes\left(q_{c, g 1}, \ldots, q_{c, g}\right)\right.\right.$, $\left.\left.\iota_{N(v+1)}^{\prime} \otimes\left(q_{\delta, g 1}, \ldots, q_{\delta, g K}\right)\right)\right\}$.

Let $f\left(y_{i g t} \mid \boldsymbol{\beta}, \zeta_{g}, \boldsymbol{\eta}_{i g}\right)$ be the density of the dependent variable in equation (2.7) which is the density of a normal with mean $\mathbf{z}_{i g t}^{\prime}\left(\boldsymbol{\beta}+\zeta_{g}+\boldsymbol{\eta}_{i g}\right)$ and variance $\sigma_{i g}^{2}$. We consider the marginal distribution $f(y \mid \boldsymbol{\beta})$ of the observable obtained by integrating out the random effects. Consider the moment generating 
function

$$
\begin{aligned}
& \int \exp \{\theta y\} f(y \mid \boldsymbol{\beta}) d y \\
& =\int \exp \{\theta y\} f\left(y \mid \boldsymbol{\beta}, \boldsymbol{\zeta}_{g}, \boldsymbol{\eta}_{i g}\right) f\left(\boldsymbol{\zeta}_{g}\right) f\left(\boldsymbol{\eta}_{i g}\right) d \boldsymbol{\zeta}_{g} d \boldsymbol{\eta}_{i g} d y \\
& \quad=\int \exp \left(\theta \mathbf{z}_{i g t}^{\prime}\left(\boldsymbol{\beta}+\boldsymbol{\zeta}_{g}+\boldsymbol{\eta}_{i g}\right)+\frac{\sigma_{i g}^{2}}{2} \theta^{2}\right) f\left(\boldsymbol{\zeta}_{g}\right) f\left(\boldsymbol{\eta}_{i g}\right) d \boldsymbol{\zeta}_{g} d \boldsymbol{\eta}_{i g} \\
& =\exp \left(\theta \mathbf{z}_{i g t}^{\prime} \boldsymbol{\beta}\right) \exp \left(\theta^{2} \mathbf{z}_{i g t}^{\prime} R \mathbf{z}_{i g t}+\theta^{2} \mathbf{z}_{i g t}^{\prime} Q_{g} \mathbf{z}_{i g t}+\frac{\sigma_{i g}^{2}}{2} \theta^{2}\right),
\end{aligned}
$$

which is the moment generating function of a normal random variable with mean $\mathbf{z}_{i g t}^{\prime} \boldsymbol{\beta}$ and variance $\sigma_{i g}^{2}+\mathbf{z}_{i g t}^{\prime}\left(R+Q_{g}\right) \mathbf{z}_{i g t}$.

Acknowledgements. This research used the SCSCF multiprocessor cluster system at University Ca' Foscari of Venice. This paper is part of the research activities at the Centre for Applied Macro and Petroleum economics (CAMP) at the BI Norwegian Business School.

We thank for their useful comments on a previous version an Associate Editor, two Referees, Sylvia Kaufmann, Dimitris Korobilis, Haroon Mumtaz, Davide Pettenuzzo, Christian Schumacher, Michael Smith, our discussant at the 2017 ASSA meeting Jonathan Wright, the participants at: the Bayesian Analysis and Modeling Summer Workshop 2015 in Melbourne, the 9th International Conference on Computational and Financial Econometrics in London, the CAMP Workshop on Commodities, business cycles and monetary policy, the 10th Annual RCEA Bayesian Econometric Workshop, the Bank of England and Essex Business School Workshop on "Time Variation and Nonlinear Models in Econometrics and Macroeconomics", the 3rd Padova Macro Talks, the 22nd Computing in Economics and Finance conference, the Bundesbank seminar, the University of Padova seminar, the 7th European Seminar on Bayesian Econometric annual meeting in Venice, the 2017 American Economic Association annual meeting, the Seventh Italian Congress of Econometrics and Empirical Economics, the EABCN Conference in Florence, the University of Essex workshop and the 4th International Association for Applied Econometrics conference. The views expressed are those of the authors and do not necessarily reflect those of the Deutsche Bundesbank.

\section{SUPPLEMENTARY MATERIAL}

Supplement B: Computational details (DOI: 10.1214/18-AOAS1168SUPPB; .pdf). This document contains the derivation of the full conditional distributions of the Gibbs sampler and a description of the sampling methods used. 
Supplement C: Simulation results (DOI: 10.1214/18-AOAS1168SUPPC; .pdf). This document reports the results of the simulation experiments used to check the efficiency of the proposed MCMC procedure.

Supplement D: Data description (DOI: 10.1214/18-AOAS1168SUPPD; .pdf). This document contains a description and a preliminary analysis of the data used in the empirical application.

\section{Supplement E: Further empirical results (DOI: 10.1214/18-} AOAS1168SUPPE; .pdf). This document provides further empirical results and robustness checks.

\section{REFERENCES}

Alessandri, P. and Mumtaz, H. (2014). Financial Regimes and Uncertainty Shocks. Working Papers No. 729, Queen Mary University of London, School of Economics and Finance.

BAKER, S. R., BLOOM, N. and DAVIS, S. J. (2016). Measuring economic policy uncertainty. The Quarterly Journal of Economics 131 1593-1636.

BASSETti, F., CASARIN, R. and Leisen, F. (2014). Beta-product dependent Pitman-Yor processes for Bayesian inference. J. Econometrics 180 49-72. MR3188911

Billio, M., Casarin, R., Ravazzolo, F. and van DiJK, H. K. (2012). Combination schemes for turning point predictions. Quarterly Review of Economics and Finance 52 402-412.

Billio, M., CASARin, R., Costola, M. and Pasqualini, A. (2016a). An entropy-based early warning indicator for systemic risk. Journal of International Financial Markets, Institutions and Money 45 42-59.

Billio, M., Casarin, R., Ravazzolo, F. and Van DiJK, H. K. (2016b). Interconnections between Eurozone and US booms and busts using a Bayesian panel Markov-switching VAR model. J. Appl. Econometrics 31 1352-1370. MR3580904

BLOoM, N. (2009). The impact of uncertainty shocks. Econometrica 77 623-685. MR2531358

Bloom, N. (2014). Fluctuations in uncertainty. Journal of Economic Perspectives 28 153-76.

Caggiano, G., Castelnuovo, E. and Groshenny, N. (2014). Uncertainty shocks and unemployment dynamics in U.S. recessions. Journal of Monetary Economics 67 78-92.

CANOVA, F. and CICCARELLI, M. (2004). Forecasting and turning point predictions in a Bayesian panel VAR model. J. Econometrics 120 327-359. MR2058892

CANOVA, F. and CicCARELLI, M. (2009). Estimating multicountry VAR models. Internat. Econom. Rev. 50 929-959. MR2542805

Carriero, A., Clark, T. E. and Marcellino, M. (2018). Measuring uncertainty and its impact on the economy. Rev. Econ. Stat. To appear.

Casarin, R., Foroni, C., Marcellino, M. and Ravazzolo, F. (2018). Supplement to "Uncertainty through the lenses of a mixed-frequency Bayesian panel Markovswitching model." DOI:10.1214/18-AOAS1168SUPPB, DOI:10.1214/18-AOAS1168SUPPC, DOI:10.1214/18-AOAS1168SUPPD, DOI:10.1214/18-AOAS1168SUPPE.

Chib, S. and Greenberg, E. (1995). Hierarchical analysis of SUR models with extensions to correlated serial errors and time-varying parameter models. J. Econometrics 68 339-360.

ClARK, T. E. and RAVAZzolo, F. (2015). Macroeconomic forecasting performance under alternative specifications of time-varying volatility. J. Appl. Econometrics 30 551-575. MR3358636

Dovern, J., Fritsche, U. and Slacalek, J. (2012). Disagreement among forecasters in G7 countries. Rev. Econ. Stat. 94 1081-1096.

Ferrara, L. and Guerin, P. (2015). What Are The Macroeconomic Effects of High-Frequency Uncertainty Shocks? Economics Working Papers No. 2015-12, Univ. Paris West-Nanterre la Defense, EconomiX. 
Foroni, C. and MARCELLINO, M. (2014). Mixed-frequency structural models: Identification, estimation, and policy analysis. J. Appl. Econometrics 29 1118-1144. MR3293137

Foroni, C., MARCEllino, M. and SchumACHER, C. (2015). Unrestricted mixed data sampling (MIDAS): MIDAS regressions with unrestricted lag polynomials. J. Roy. Statist. Soc. Ser. A 178 57-82. MR3291761

Foroni, C., RaVAzzolo, F. and Ribeiro, P. J. (2015). Forecasting commodity currencies: The role of fundamentals with short-lived predictive content. Working Paper No. 2015/14, Norges Bank.

FRÜHWIRTH-SCHNATTER, S. (2006). Finite Mixture and Markov Switching Models. Springer, New York. MR2265601

Ghysels, E., Santa-Clara, P. and Valkanov, R. (2005). There is a risk-return trade-off after all. J. Financ. Econ. 76 509-548.

Ghysels, E., Sinko, A. and Valkanov, R. (2007). Midas regressions: Further results and new directions. Econometric Rev. 26 53-90. MR2339264

GuÉRIN, P. and MARCELlino, M. (2013). Markov-switching MIDAS models. J. Bus. Econom. Statist. 31 45-56. MR3030797

Jurado, K., Ludvigson, S. C. and NG, S. (2015). Measuring uncertainty. Am. Econ. Rev. 105 $1177-1216$

KAUFMANN, S. (2010). Dating and forecasting turning points by Bayesian clustering with dynamic structure: A suggestion with an application to Austrian data. J. Appl. Econometrics 25 309-344. MR2758637

KAUFMANN, S. (2015). $K$-state switching models with time-varying transition distributionsdoes loan growth signal stronger effects of variables on inflation? J. Econometrics 187 82-94. MR3347296

Khalaf, L., Kichian, M., Saunders, C. and Voia, M. (2013). Dynamic Panels with MIDAS Covariates: Estimation and Fit Technical Report.

Koop, G. and KorobiLis, D. (2016). Forecasting With High Dimensional Panel VARs. Strathclyde Business School Working Paper.

LIU, J. S. (1994). The collapsed Gibbs sampler in Bayesian computations with applications to a gene regulation problem. J. Amer. Statist. Assoc. 89 958-966. MR1294740

Ludvigson, S. C., MA, S. and NG, S. (2015). Uncertainty and Business Cycles: Exogenous Impulse or Endogenous Response? NBER Working Papers No. 21803.

Magnus, J. R. and Neudecker, H. (1999). Matrix Differential Calculus with Applications in Statistics and Econometrics. Wiley, Chichester. MR1698873

Min, C. and ZELLNER, A. (1993). Bayesian and non-Bayesian methods for combining models and forecasts with applications to forecasting international growth rates. J. Econometrics 56 89-118.

Mumtaz, H. and TheOdoridis, K. (2018). The changing transmission of uncertainty shocks in the US. J. Bus. Econom. Statist. 36 239-252. MR3790211

Pettenuzzo, D., Timmermann, A. and Valkanov, R. (2016). A MidAS approach to modeling first and second moment dynamics. J. Econometrics 193 315-334. MR3515896

Robert, C. P. and Casella, G. (1999). Monte Carlo Statistical Methods. Springer, New York. MR1707311

RoBERTS, G. O. and SAHU, S. K. (1997). Updating schemes, correlation structure, blocking and parameterization for the Gibbs sampler. J. Roy. Statist. Soc. Ser. B 59 291-317. MR1440584

Rodriguez, A. and Puggioni, G. (2010). Mixed frequency models: Bayesian approaches to estimation and prediction. Int. J. Forecast. 26 293-311.

Rossi, B. and SEKHPOSYAN, T. (2015). Macroeconomic uncertainty indices based on nowcast and forecast error distributions. Am. Econ. Rev. 105 650-655.

ScotTI, C. (2016). Surprise and uncertainty indexes: Real-time aggregation of real-activity macrosurprises. Journal of Monetary Economics 82 1-19. 
R. CASARIN

DEPARTMENT OF ECONOMICS

UNIVERSITY CA' FosCARI OF VENICE

SAN GIOBBE 873/B

30121 VENEZIA

ITALY

E-MAIL: r.casarin@unive.it

M. MARCELLINO

DEPARTMENT OF ECONOMICS

BOCCONI UNIVERSITY

VIA ROENTGEN 1

20136, MiLANO

ITALY

E-MAIL: massimiliano.marcellino@unibocconi.it

\section{FORONI}

DEUTSCHE BUNDESBANK

WILHELM-EPSTEIN STR. 14

60431 FranKFURT AM MAIN

GERMANY

E-MAIL: claudia.foroni@bundesbank.de

F. RAVAZZOLO

FACULTY OF ECONOMICS

UNIVERSITY OF BOZEN/BOLZANO

PIAZZA UNIVERSITÀ, 1

39100 BOLZANO

ITALY

E-MAIL: francesco.ravazzolo@unibz.it 